\title{
Statistical Analysis of the equilibrium configurations of W7-X stellarator
}

\author{
A.Sengupta ${ }^{1}$, J.Geiger ${ }^{1}$, P.J.Mc Carthy ${ }^{2}$ \\ ${ }^{1}$ Max-Planck-Institut für Plasmaphysik, Euratom Association, Greifswald, Germany \\ ${ }^{2}$ Dept. of Physics, University College Cork, Association EURATOM-DCU, Cork, Ireland
}

\begin{abstract}
Equilibrium magnetic configurations of W7-X stellarator plasma were analysed in this study. The statistical method of Function Parametrization was used to recover the physical properties of the magnetic configurations, such as the flux surface geometry, the magnetic field, the iota profile, etc, from simulated experimental data. The study was carried out with a net toroidal current. Idealized "measurements" were first used to recover the configuration. These "measurements" were then perturbed with noise and the effect of this perturbation on the recovered configuration parameters was estimated. The noise was scanned over a range large enough to encompass that expected in the actual experiment. In the process, it was possible to ascertain the limit of tolerable noise that can be allowed in the inputs so as not to significantly perturb the outputs recovered with noiseless "measurements". Generally, a cubic polynomial model was found to be necessary for noise levels below $10 \%$. For higher noise levels, a quadratic polynomial performed as well as the cubic. The noise level of $10 \%$ was also the approximate limit up to which the recovery with ideal measurements was generally reproduced. For the flux geometry recovery, however, the quadratic model performed similar to the cubic for any value of noise, with the latter model proving to be significantly better only for the noiseless case. Also, with noisy predictors the recovery error for the flux surfaces increases linearly with effective radius from the plasma core up to the edge.
\end{abstract}




\section{Introduction}

W7-X $[1,2]$ is a fully optimized stellarator of the Helias type, with a five-fold toroidal symmetry (i.e., five toroidal periods), under construction at the Max-Planck Institut für Plasmaphysik (IPP), Greifswald, Germany. It has an average major radius of $5.5 \mathrm{~m}$ and an average minor radius of $55 \mathrm{~cm}$, giving an aspect ratio of about 10 . A set of $2 \times 5$ modular field coils (MFC) in each period produces the standard configuration with a rotational transform $t_{b}=1$ at the boundary with five surrounding natural islands forming a separatrix. The value of $t_{b}$ can be varied between 5/6 (low iota case) and 5/4 (high iota case) using $2 \times 2$ additional planar coils (PLC) per period. There are a total of 10 control coils (two coils per period) which, however, do not have any role in the generation of the magnetic configuration and are basically for controlling the magnetic islands at the boundary. The helical magnetic axis is pentagon-shaped when viewed from the top. The plasma has a bean-shaped cross section at the corners of the pentagon where the magnetic field is the strongest to allow for the trapping of fast particles within the straight portions. Within a half-period, the plasma cross section varies from a bean-shape $(\phi=$ 0 degree), through a tear-drop shape ( $\phi=18$ degrees) to a triangular shape ( $\phi=36$ degrees).

An important goal of $\mathrm{W} 7-\mathrm{X}$ is to investigate the steady state capabilities of fusion devices. For stellarators this essentially implies a real time monitoring of the discharges which have long pulse lengths, of the order of minutes. For a real time study one must have means to generate a magnetic configuration in some fraction of a second, while 3-D magnetohydrodynamic (MHD) equilibrium codes, which simulate stellarator configurations, do so in minutes to hours depending on the resolution and available computational resources. This essentially requires the use of methods which are fast and accurate.

For W7-X we have planned a sequence of in-depth analyses of the magnetic configurations which, ultimately, will lead to a proper understanding of plasma equilibrium, stability and transport. The first step in that sequence involved a study of the W7-X vacuum configurations with magnetic islands [3] where we used the statistical, inverse mapping method of Function Parametrization (FP) [4 - 6] to recover the physical properties of the configurations. Due to its speed, this method is useful in a real time monitoring, control and data analysis where existence of MHD equilibrium is not necessary. Our experience in [3], where an Artificial Neural Network technique did not improve upon the results of a cubic polynomial FP model, encouraged us to use FP again.

This paper reports the results on the next step of analysis - the scenario at finite $\beta$ - where there is a full MHD equilibrium. Magnetic configurations in presence of plasma pressure are important because of significant changes to the vacuum flux surface topology at finite beta. With partial optimization, meaning a reduction of plasma Pfirsch-Schlüter currents compared to a classical stellarator, the previous stellarator in operation at IPP, W7-AS [7], was able to achieve a volume-averaged beta $(\langle\beta>)$ value of up to $3.4 \%$ with a magnetic field of 1 Tesla. $\mathrm{W} 7-\mathrm{X}$ is fully optimized in the sense that the plasma influence on the magnetic configuration has been strongly reduced by the minimization of bootstrap currents (except for low-mirror configurations) and Pfirsch-Schlüter currents. This provides good MHD stability properties up 
to $\langle\beta\rangle=5 \%$. However, in spite of the optimization, the bootstrap current does not vanish completely. Up to $\pm 50 \mathrm{kA}$ of residual current is expected, depending on the configuration and the plasma parameters, with a strong influence on the island divertor performance determined by the location of the separatrix formed from the boundary islands. Electron Cyclotron Current Drive (ECCD) and Neutral Beam Current Drive (NBCD) are, therefore, envisaged to control the total plasma current $I_{p}$ for proper divertor operation. Additionally, to show the reactor capabilities of stellarators quasi-isodynamicity evolving at finite-beta provides good fast particle confinement with increasing beta [2].

For the present study we neglected the presence of magnetic islands in the equilibrium configurations. Islands in finite $\beta$ conditions can be simulated only by codes like HINT [8] or PIES [9]. However, these codes are computationally very demanding due to the complexity of the calculations they have to perform, so the possibility of using them to generate large databases for statistical analyses is still very bleak. Furthermore, the external coils of W7-X were assumed to be accurately positioned, and error fields arising from, e.g., coil misalignments, were not included in our statistical model.

The paper is outlined as follows. Section 2 describes the database generation with the equilibrium code. Section 3 informs the reader of the decision on the choice of the actual inputs for our statistical model. Section 4 discusses the statistical approach used in the work. Section 5 describes the scheme of adding measurement noise, while Section 6 shows the results in all their detail. The seventh and final section summarizes the paper.

\section{Details of database generation}

Simulated W7-X plasmas were produced by VMEC2000, a 3-D equilibrium code [10] that assumes nested flux surfaces, thereby neglecting magnetic islands as mentioned in the previous section. The geometry and the magnetic field on the flux surfaces are given as Fourier coefficients (FC's) with a modest number of harmonics. A database of about 8000 such configurations was generated for the statistical analysis.

The parameters which were varied randomly and independently consist of the six external coil current ratios $i_{2}, \ldots, i_{5}, i_{A}, i_{B}$ (formed by normalizing the absolute currents $I_{1}, \ldots, I_{5}$ (Modular field coils, MFCs), $I_{A}$ and $I_{B}$ (Planar field coils, PFCs) to $I_{1}$ ), the parameters of the profiles (as functions of normalised toroidal flux $s$ ) of plasma pressure and the toroidal plasma current (four parameters for each profile), and finally the plasma size (represented by the effective minor radius of the boundary surface, $a_{e f f}$ ) which is required to vary the plasma volume. This resulted in a total of $N_{m}=15$ parameters, thereby yielding a higher dimensional parameter space for database generation than that used for a similar investigation [11] for the W7-AS stellarator where a vanishing toroidal current profile was assumed.

As in [11] we aim at a global FP over the entire parameter range of the 15 parameters. Therefore we use the strategy of randomly varying the parameters to build up the database for the regression which has the advantage that a database of $N$ cases has $N$ distinct values for 
every parameter varied. This is far more efficient than generating cases on a regularly spaced grid where, for a cubic polynomial model, the absolute minimum number of cases, namely, involving four gridpoints for each of the 15 input parameters, would be an impractically large $4^{15} \simeq 10^{9}$.

The plasma parameters were varied to allow a good FP for their expected values in W7-X: volume-averaged $<\beta>$ of up to $5 \%$ and a net toroidal current of up to $\pm 50 \mathrm{kA}$ for a mean field strength of about 2 Tesla throughout the database. In addition, the same parameter space was retained for the coil current ratios as was used in [3], namely, $i_{2}, \ldots, i_{5}$ in the range $[0.6,1.2]$ and $i_{A}, i_{B}$ lying within $[-1,1]$.

The profiles of pressure

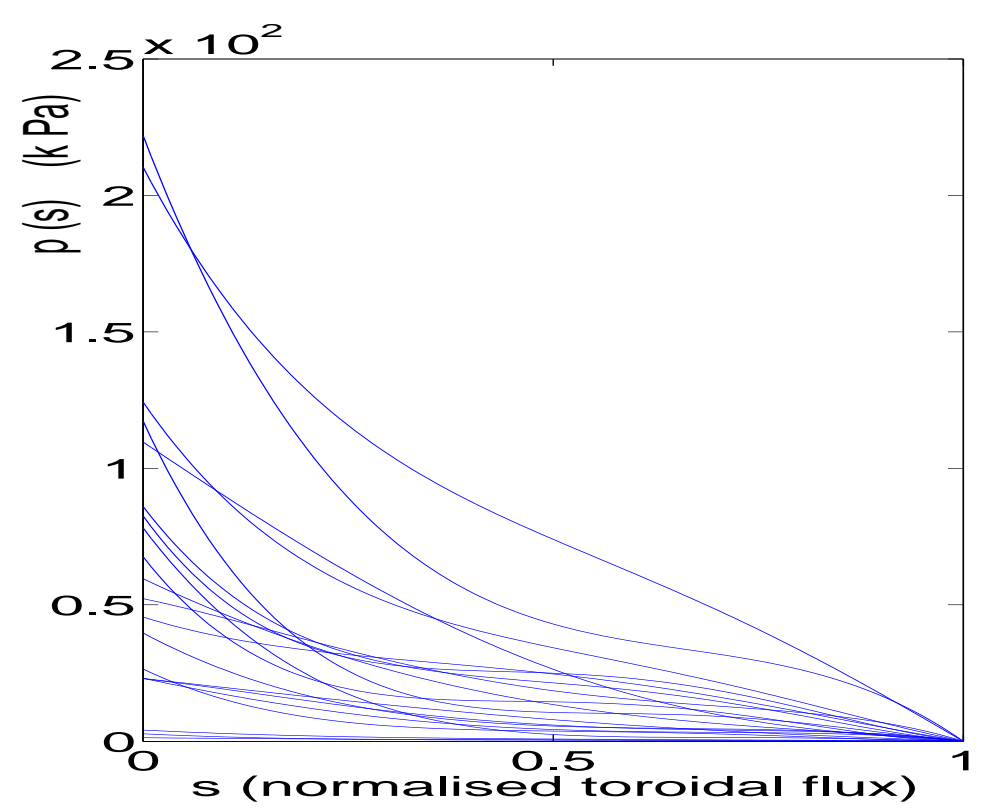

Figure 1: A sample of plasma pressure profiles from the database. and plasma current were chosen as a sequence of polynomials in the forms

$$
\begin{aligned}
& p(s)=\sum_{i=1}^{n} a_{i} b_{i}(s) \\
& I(s)=\sum_{i=1}^{n} c_{i} d_{i}(s),
\end{aligned}
$$

respectively, where $b_{i}(s)$ and $d_{i}(s)$ are polynomials of degree $i$ in the normalized toroidal flux $s$. We chose $n=4$ in both cases. These polynomials are given by

$$
\begin{array}{ll}
b_{1}(s)=1-s & d_{1}(s)=s \\
b_{2}(s)=\left(1-4 s+3 s^{2}\right) & d_{2}(s)=s(1-s) \\
b_{3}(s)=s(1-s)(1-2 s) & d_{3}(s)=s(1-s)(1-2 s) \\
b_{4}(s)=s(1-s)\left(1-5 s+5 s^{2}\right) & d_{4}(s)=s(1-s)\left(1-5 s+5 s^{2}\right) \\
\text { with } \int_{0}^{1} b_{1}(s) d s=0.5 \text { and } \int_{0}^{1} b_{i}(s) d s=0 \text { for } i \geq 2 .
\end{array}
$$

Only the first polynomial $b_{1}(s)$ contributes to $\int p(s) d s$, thus relating $a_{1}$ to the volumeaveraged pressure, while $b_{2}(s)$ allows for pressure peaking variation which is inferred from $a_{1}$ and $a_{2}$. For the current profile, $d_{1}(s)=s$ is the only term contributing at the plasma edge, hence $c_{1}$ equals the total plasma current $I(s=1)$. The higher order polynomials were constructed so as not to alter the physics contributions of the lower orders. Figure 1 shows some of the typical pressure profiles used in the analysis. Control of the bootstrap current by ECCD or NBCD leads to quite different toroidal current profiles as shown in Fig. 2.

The criteria for deciding upon the usable cases for analysis were: 
(a) Convergence of the code;

(b) Monotonically decreasing pressure profiles, i.e., $d p / d s<0$.

(c) $t_{0} \geq 0.16$;

Too small values of $t_{0}$ result in large Shafranov shift of the magnetic axis if there is a strong pressure gradient in the low-iota region, so we impose a lower limit.

(d) $0.62 \leq t_{b} \leq 1.32$;

This places a constraint on the toroidal plasma current, as too large currents may deteriorate the flux surfaces at the boundary.

(e) Restricting $\beta_{0} \leq 12 \%$ ( $\beta_{0}$ is the plasma $\beta$ on the magnetic axis);

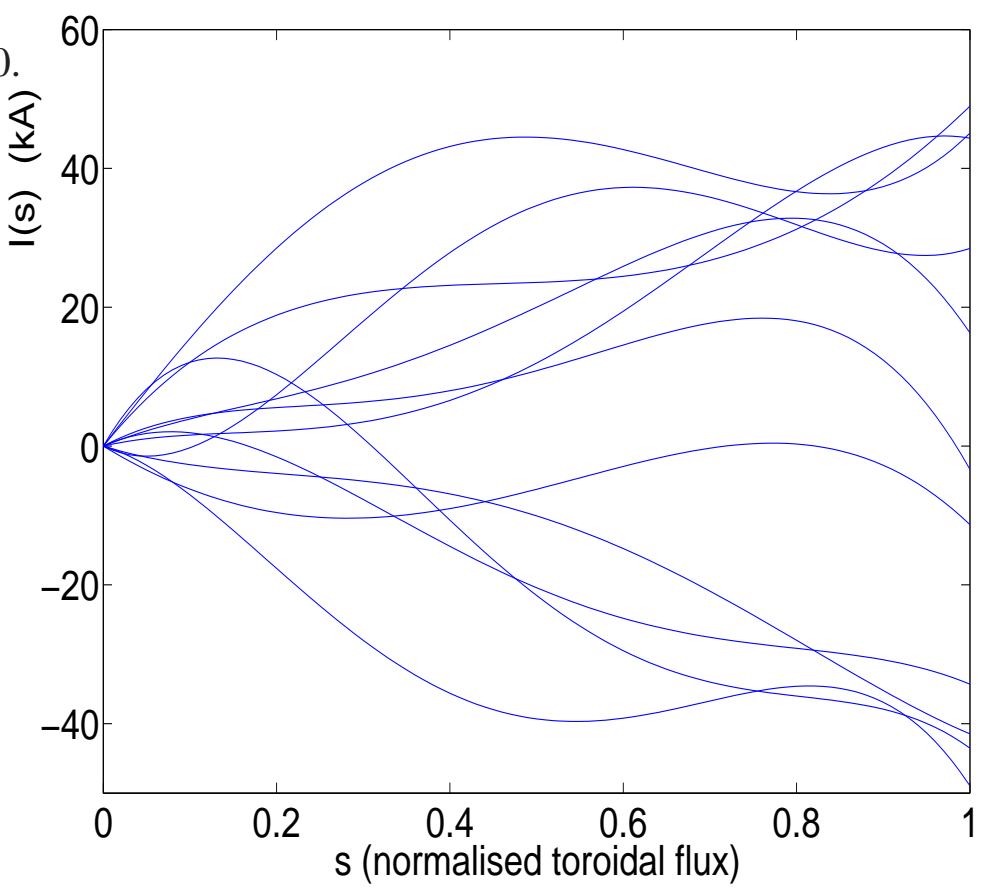

Figure 2: A sample of toroidal plasma current profiles from the database.

This constraint restricts the peaking of the pressure profile for large $\langle\beta>$-values. The practical reason for this is to get converged VMEC-equilibria within a reasonable computational effort. Without this constraint, we would calculate equilibria with $\beta_{0}$ up to $30 \%$. The physical reasoning is that the experimental scenarios for high $-\langle\beta>$ are expected to be at low magnetic field strengths ( $B=0.8-1.3$ Tesla) with Neutral Beam Injection (NBI)-heating leading to broad pressure profiles. For W7-AS the peaking factor in such discharges was $\sim 2$. In contrast, Electron Cyclotron Resonance (ECR)-heated plasmas (140GHz, X2-mode), which may show very peaked temperature profiles resulting in peaked pressure profiles, correspond to magnetic fields of 2.5 Tesla where lower $\langle\beta>$-values are predicted because of the peaking and the additional constraint due to the cutoff-density.

(f) $a_{\text {eff }} \leq 60 \mathrm{~cm}$, as it is unlikely to exceed $55 \mathrm{~cm}$ in experiments.

Figure 3 shows the configuration space in the $i_{A}-i_{B}$ plane. The void at the lower left corner is caused by restrictions (d) corresponding to the high- $t$ region. In view of the positive shear of the $t$ profiles for larger $r_{e f f}$, restriction (f) also plays some role. The points in the rest of the space are more or less uniform.

The basic plasma parameters chosen for the recovery were the profiles (as functions of an effective flux surface radius $r_{e f f}$ ) of $t$ and the FC's of the magnetic field strength $\left(B_{m n}\right)$, the geometry $\left(R_{m n}, Z_{m n}\right)$ and the periodic renormalization function $\left(\lambda_{m n}\right)$, where $m$ and $n$ are, respectively, the poloidal and the toroidal Fourier mode number. The quantity $r_{\text {eff }}$ for each flux surface is defined as the minor radius of a torus with circular cross section, having the same major radius and the same volume. The parameter $\lambda$ facilitates rapid convergence of the Fourier series of the flux surface geometry and also modifies the poloidal angular coordinate 
$\left(\theta^{*}=\theta+\lambda\right)$ in order that the field lines are straight in the $\left(\theta^{*}-\phi\right)$ coordinate system [12].

In figure 4 we have plotted a few typical profiles of $t$ from the database, for different configurations with different pressure and toroidal current profiles showing the resulting variety which is mainly due to the toroidal current profile variation. This prime dependency is shown again in section 6(b) and figure 14.

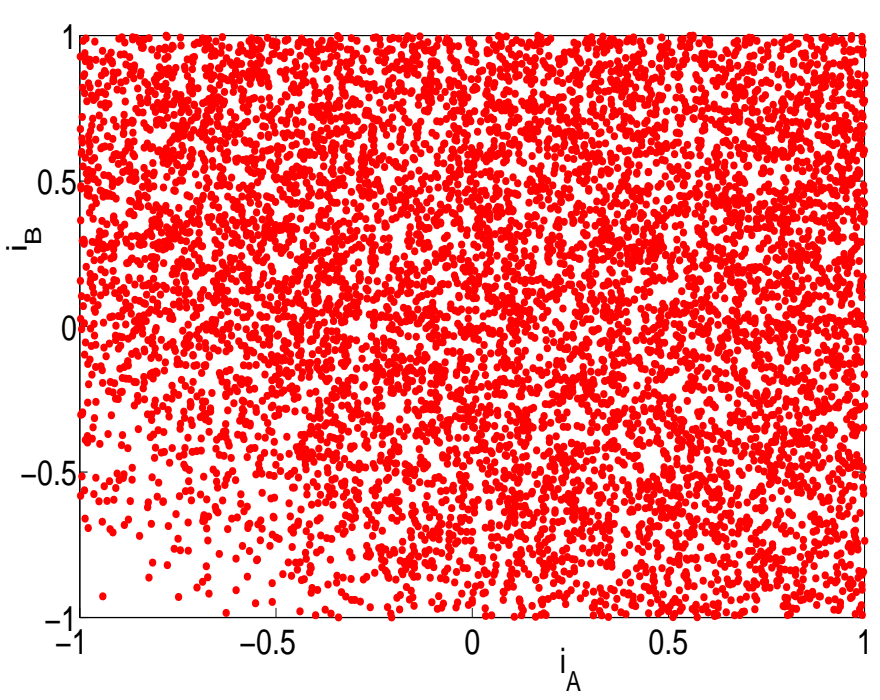

\section{Choice of predictors for Figure 3: The configuration space for the planar coil current FP model ratios $i_{A}$ and $i_{B}$. The void (lower left corner) is caused mainly by restriction $(d)$ corresponding to high-t operation.}

In our (simulated) experiment with non-magnetic "measurements", we assumed knowledge of the external coil currents, the plasma size parameter $a_{e f f}$, and the profiles of plasma pressure and the toroidal plasma current. Once a database containing these "measurements" was generated, it was necessary to decide upon the predictors, or inputs, we would be using for the statistical FP model for equilibrium reconstruction. For the coil currents, the chosen inputs were the current ratios, as already described in Section 2. The plasma size $a_{e f f}$ formed an input itself. For the plasma pressure and toroidal current data, the independently- generated coefficients $a_{1}-a_{4}$ and $c_{1}-c_{4}$ in equations (1) and (2), respectively, were possible candidates to be the model inputs, and were actually used as predictors in an earlier analysis [13].

However, the actual set of predictors decided for the statistical model in this study did not explicitly contain the coefficients $a_{1}-a_{4}$ and $c_{1}-c_{4}$. Their inclusion involves the problem of having to derive them from (noisy) experimental pressure and toroidal current profile data. Since the coefficients of the higher order polynomials become increasingly sensitive to noise, the quality of plasma parameter recovery rapidly worsens with increase of noise level. In fact, in an exploratory analysis we found that the regression completely fails for noise levels $\geq 20 \%$ for all the output plasma parameters, with the exception of the $m=0$ Fourier coefficients. That is why we chose the more robust approach of a Principal Component Analysis (PCA) of the profile data, and the significant principal components (PC's), meaning those PC's with significant eigenvalues or variance, were the inputs to our model.

The actual PCA was carried out on the noiseless profile data, and the corresponding eigenvectors of the significant PC's were stored. Later on, the noisy PC's were calculated by a linear combination of the stored (noiseless) eigenvectors with the noisy profile data. The advantages of this method are the following:

(i) although the PC's with decreasing eigenvalues also become increasingly noisy, they do 
not show the same strong sensitivity to noise when derived from noisy profile data;

(ii) a PCA of the profile data can be carried out for any functional form of the profile.

The PCA of the profile data showed that the first four PC's accounted for the entire $100 \%$ of the radial variation for both the plasma pressure and the current. This was the expected result, because the simulated profile data were generated from the parametrizations (1) and (2), where the profile variables $p(s)$ and $I(s)$ vary linearly with the coefficients $a_{1}-a_{4}$ and $c_{1}-c_{4}$, respectively.

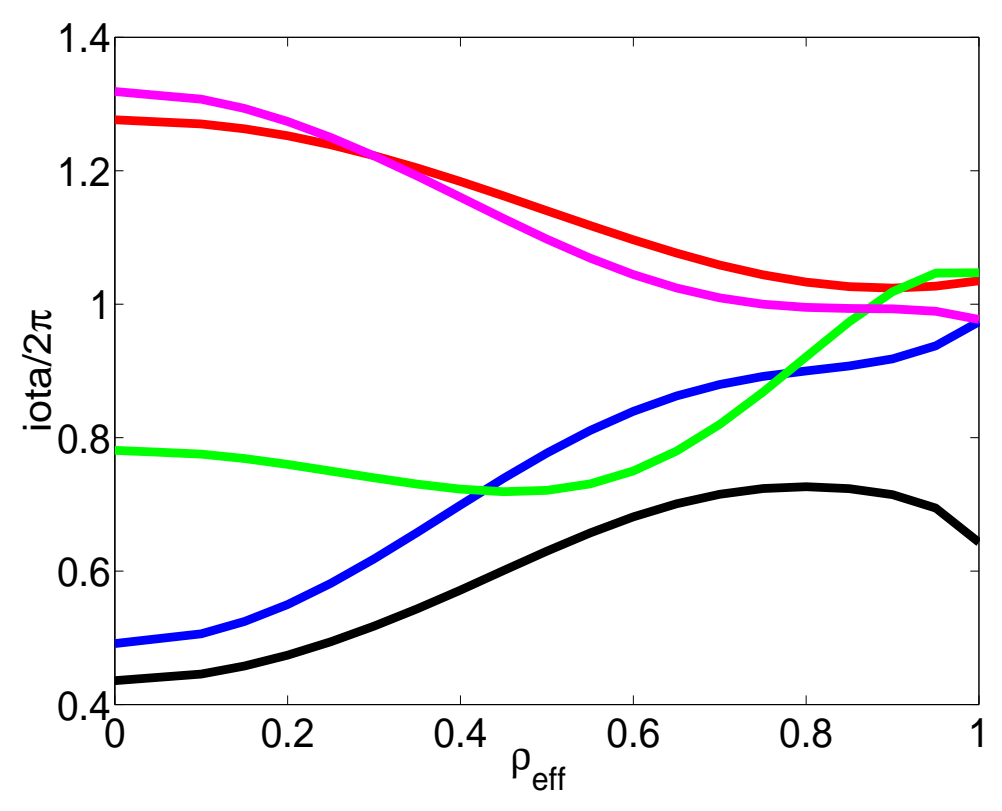

Figure 4: A sample of typical iota profiles from the database, for different configurations. Observed variations in the iota profiles are primarily due to the different current profiles.

\section{Selection of the FP model}

In this study we chose to recover the physical properties of the plasma magnetic configuration in the form of radial profiles of different parameters, so our statistical model should incorporate this radial behaviour. In [11] the output profile parameters, such as the FC's and the rotational transform, were compactly parameterized as radial polynomials of the form

$$
f\left(x_{j}, r_{e f f}\right)=p_{0}+p_{1} r_{e f f}+p_{2} r_{e f f}^{2}+\ldots
$$

where each of the coefficients $p_{0}, p_{1}$, etc, involved a quadratic combination of the independent input parameters, or predictors, $x_{j}, j$ ranging from 1 to $N_{m}$. Even in [3] where cubic combinations of the inputs were found to be necessary, a model of the form of equation (3) could still be used as $N_{m}(=6)$ was small. However, with $N_{m}=15$ predictors used in the present study, each of the above coefficients now contains $\frac{\left(N_{m}+1\right)\left(N_{m}+2\right)}{2}=136$ quadratic, or $\frac{\left(N_{m}+1\right)\left(N_{m}+2\right)\left(N_{m}+3\right)}{6}=816$ cubic combinations. For the latter case, this involves the calculation of thousands of coefficients in the model (3). Thus, the radial polynomial approach becomes unrealistic for the present study.

Instead, the following method was used for the recovery of the plasma profile parameters. A PCA of the profile parameters, valued at $N_{\text {rad }}=21$ radial points equidistant in $r_{\text {eff }}$, was carried out. The 21 radial points were then replaced by $n_{S}(<<21)$ significant PC variables (SPCV) $y_{\ell}\left(\ell=1, \ldots, n_{S}\right)$, which may be interpreted as radial moments of the profile with the weighting given by the leading $n_{S}$ eigenvectors. The eigenvectors are derived from the $21 \times 21$ covariance matrix of the raw profile parameters and play the role of radial eigenfunctions. which 
are determined by the leading $n_{S}$ eigenvectors (playing the role of radial eigenfunctions) of the $21 \times 21$ covariance matrix of the raw profile parameters. These $y_{\ell}$ formed the response variables for the regression. Each $y_{\ell}$ was calculated from the projection of the 21-element radial vector along the $\ell^{t h}$ principal component direction.

The model to recover the $n_{S}$ independent SPCV of the radial profile for each plasma parameter consisted of a "mixed" quadratic (q-FP)

$$
y_{\ell}=\sum_{i=0}^{N_{m}} \sum_{j=0}^{i} p_{i j} x_{i} x_{j}
$$

or "mixed" cubic (c-FP)

$$
y_{\ell}=\sum_{i=0}^{N_{m}} \sum_{j=0}^{i} \sum_{k=0}^{j} p_{i j k} x_{i} x_{j} x_{k}
$$

polynomials in the predictors $x$. The number of model coefficients for scalar parameters, for $N_{m}=15$, were 136 for q-FP and 816 for c-FP, which is the same as the number of quadratic and cubic combinations of the $N_{m}$ independent predictors as shown earlier. Thus, with about 5000 cases chosen for "training" there was a sufficient number of degrees of freedom in both models for a reliable fitting.

The regression was then tested on a separate test dataset of about 2400 observations, drawn randomly from the same 15-D configuration space as that used for training. The model coefficients, determined from the training dataset, were combined with the (quadratic or cubic) combinations of the predictors in the test dataset to recover the radial SPCV of the plasma profile variables in this dataset. The recovered SPCV $y_{\ell}^{(r e c)}$ were then linearly combined with the radial eigenvectors to get the recovered 21 -element radial vector $\bar{f}^{r e c}$ for a plasma parameter:

$$
\bar{f}^{r e c}=\sum_{\ell=1}^{n_{S}} y_{\ell}^{(r e c)} \bar{e}_{\ell}
$$

where, $\bar{e}_{\ell}$ is the $l^{\text {th }}$ eigenvector of the $21 \times 21$ covariance matrix. Finally the recovered plasma parameters were compared with those stored in the test dataset to determine recovery statistics. In Section 6 later, all the results shown are from the test dataset.

The training and the test parts were initially carried out with idealized predictors, i.e., predictors corresponding to ideal "measurements". They were further repeated with various levels of random noise in the "measurements". The noise treatment is described in the next section.

\section{Noise in "measurements"}

Recovery of our magnetic configuration was first carried out with a regression model with exact, or noiseless, predictors. However, in order to ensure a stable model, and also one that is compatible with an actual experiment, it was decided to perturb the "measurements" with noise, or measurement errors, of various levels which would encompass those expected in the experiment. These errors were assumed to have a uniform distribution whose standard deviation 
was chosen as a percentage of the root-mean-square (rms) values of the "measurements" over the database. Noise was self-consistently propagated into the predictors of the model (discussed in section 3) wherever they were derived from these "measurements".

For the recovery of the parameters of the plasma magnetic configuration, the following noise scheme was used to perturb the "measurements". The relative accuracy of the coil current measurement in W7-X has been estimated at $0.02 \%$ of $20 \mathrm{kA}$, that corresponds to a measurement error of 4 amps [3]. This, by any standards, implies a very accurate measurement, given that the coil currents will be typically of the order of tens of kiloAmps. With the rest of our "measurements" likely to be very noisy in experiments, it should suffice to leave the coil currents at their exact values. Nevertheless, we chose to add a small error to the currents before they were normalized to the current ratios. The error, quantified as $0.1 \%$ of the database rms values of the currents, was 11 amps for the modular field coils and 7 amps for the planar coils. This level of noise in the coil currents was kept constant throughout the "experiment".

For the toroidal current profile $I(s)$, we took account of the fact that, with the exception of the plasma current at the boundary, it is the current density profile, $J(s)$, which is the known quantity (from transport analysis, including heat deposition profiles) with all its uncertainties. Simulated $J(s)$ values were obtained by finite differencing the database $I(s)$ profile. The noise added to $J(s)$ was assumed

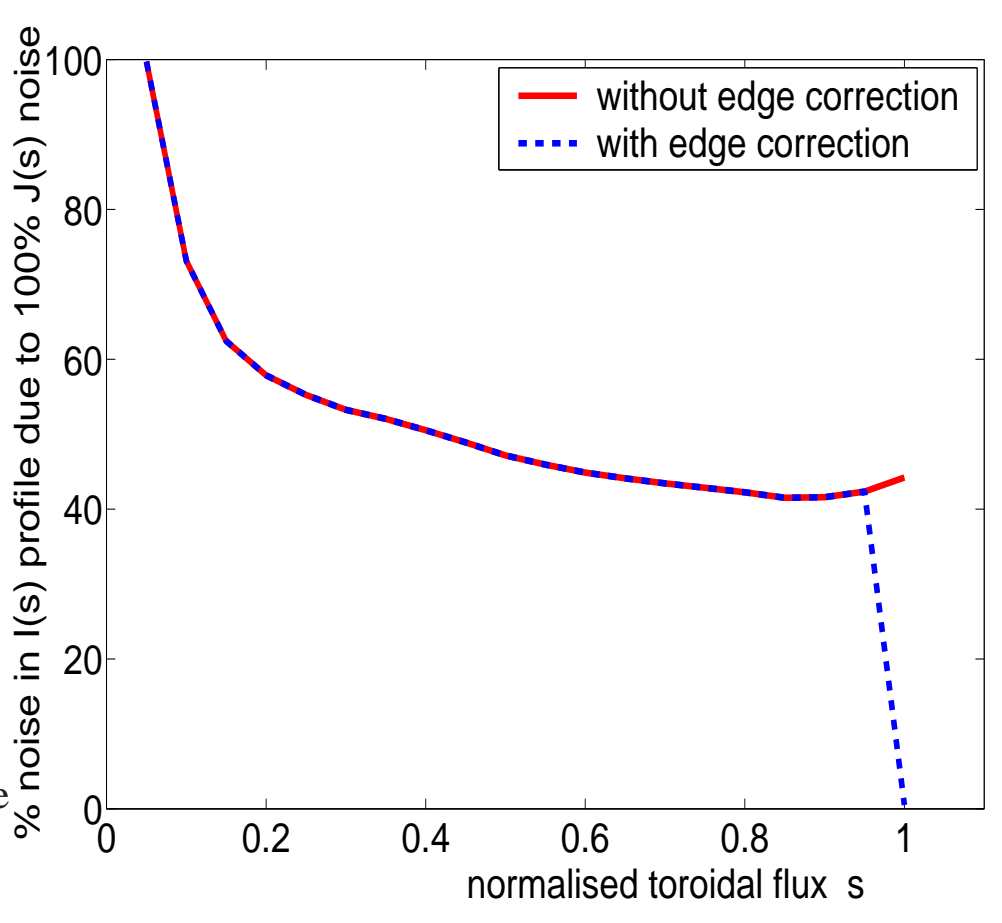
to be $x \%$ of the database Figure 5: Variation of percentage noise in I $(s)$ profile with normalrms (flux-dependent) val- ized toroidal flux s, for $100 \%$ noise in $J(s)$ profile. red curve: without ues of $J(s)$. Thus, the edge correction; blue curve: with edge correction. relative noise $(x \%)$ was uniform across the profile. The chosen values of $x$ consisted of a quasicontinuous scan from 1 to 50. However, the absolute noise varied with the flux coordinate $s$ in the same way as the database rms of $J(s)$. The noisy $\tilde{J}(s)$ profile was summed to generate a noisy $\tilde{I}(s)$ profile. Clearly, there will be noise cancellation when summing $\tilde{J}(s)$.

In the case of the edge value of the toroidal plasma current profile $I_{p}=I(s=1)$, an accurate measurement will be available from a Rogowsky coil, the error level for which has already been estimated. Thus, the noisy $\tilde{I}(s)$ input information is a combination of 


$$
\tilde{I}_{j}\left(s_{k}\right)=\sum_{k=1}^{j} \Delta s \tilde{J}\left(s_{k}\right)
$$

(where $j \leq 20$ ) and the Rogowsky coil signal $I_{p}$.

For W7-X the accuracy of $I_{p}$ is expected to be better than 50 amps for a discharge lasting for 30 minutes. With a maximum current of $50 \mathrm{kA}$ chosen for this study, this corresponds to a random error of $0.1 \%$. A further $0.5 \%$ systematic error on the chosen maximum value of $I_{p}$ may result due to a possible lack of homogeneity in the Rogowski coil winding. These errors were added in sequence on the noiseless value of $I(s=1)$. These errors are very small and, like those on the coil currents, their level was held constant. With the noisy $I(s)$ profile, including the edge value, having thus been determined, it was then combined with the stored eigenvectors to form four significant (noisy) PC's forming the predictor set for $I(s)$ as already explained in section 2 .

Figure 5 shows, as an example, the profile of the percentage noise in $I(s)$ resulting from $100 \%$ noise in $J(s)$. The red curve shows the profile without the edge correction for $I(s)$. As the limit of the integration of $J(s)$ increases, i.e., moving from the axis outwards, noise cancellation leads to decreasing noise levels in $I(s)$. However, there is some saturation within the outer $40 \%$ of the profile. Nevertheless, the noise level does come down to $\sim 43 \%$ in the plasma edge region. This gain is further enhanced by the independent and accurate measurement of the edge plasma current, as shown by the blue (dotted) curve. The localization of the edge correction is obviously due to the fact that only the total plasma current, rather than the internal distribution, is measured and its accuracies estimated. Figure 5 may be interpreted as follows. The drop in the $I(s=1)$ error introduces a shielding current density close to and inside the $s=1$ surface, the plasma boundary. This current density appropriately corrects the total plasma current and also allows an uncorrelated variation of the inner current density by providing a shielding effect. This picture gives an extreme case of the relative behaviour of the plasma current errors on the boundary and inside.

For the pressure data a basically similar scheme was followed, the noise being $y \%$ of the (database flux dependent) mean value of the pressure. The relative noise was, once again, uniform at $y \%$ along the profile. The absolute noise varied with $s$ as the mean pressure did. However, the chosen values of $y$ ranged from 0 only up to $20 \%$ as it is usually anticipated that the plasma pressure is more accurately determined than the current density. The noise scan for $a_{e f f}$ was limited to $10 \%$ of the database mean value. The noise scan was done so that, when the $J(s)$ noise was $x \%$, noise on pressure data was $(x / 2.5) \%$ and that on $a_{e f f}$ was $(x / 5) \%$. In this way several combinations of noise levels on these "measurements" can be worked with for the recovery of the magnetic configuration and, hopefully, the noise range on each of them would encompass those expected in the real experiment. Subsequently in this section and also in the figures, we would quote the $J(s)$ noise only.

This correlation of the noise levels in $J(s), p(s)$ and $a_{e f f}$ does not result in a loss of generality. Each triplet of noise levels is meant to show the quality of recovery when these measurements are perturbed by the respective levels of noise. 
Figure 6 shows the distribution of the absolute noise values for $J(s)$ and $p(s)$ over the profile in $s$. The figure actually shows the values for $100 \%$ noise as an example. Since the noise was calculated as a fixed percentage of the database (s-dependent) rms value of $J(s)$ (which equals the standard deviation $\sigma_{J}$ of $J(s)$ ) and mean value of $p(s)$, these curves also show the variation, with $s$, of $\sigma_{J}$ (upper panel) and the mean value of $p(s)$ (lower panel). The $J$-curve shows the large (and similar) variation of the plasma current density in the plasma core and the edge regions, and the much smaller, virtually constant, variation in between.

\footnotetext{
6. Results

a) Recovery of magnetic configuration.

As reported in section 4 , the recovered plasma parameters were obtained from regressions using, as response variables for the model, the significant $n_{S}$ radial SPCV of the profile variables. Table 1 shows the values of $n_{S}$ required to account for (most of) the total variance
} of the output profile vari-

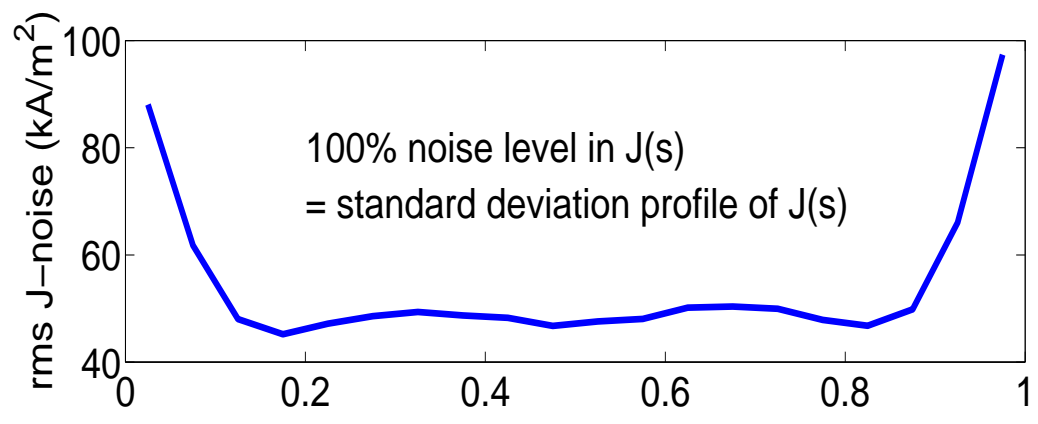
ables. For the FC's, however, there is a slight overestimation for the low-order of their database rms values.

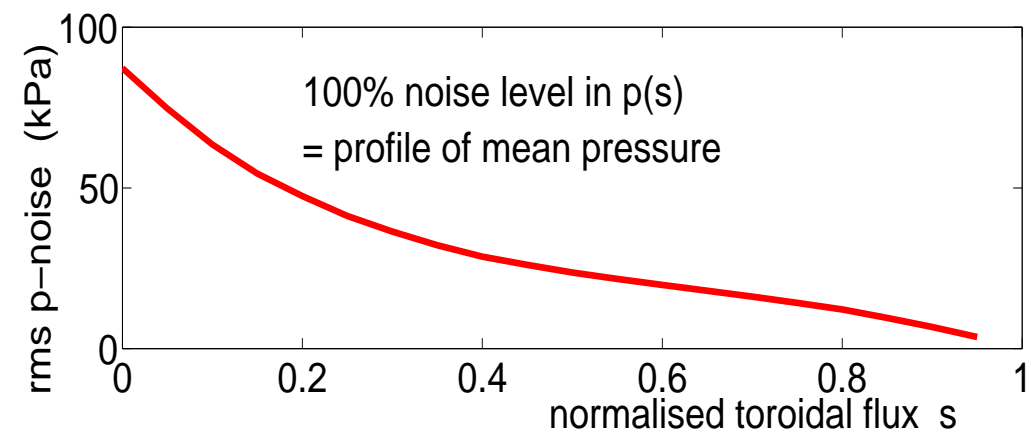

Figure 6: Variation of absolute noise in the profiles of $J(s)$ and $p(s)$ corresponding to a fixed percentage (the figure is for 100\%) Fourier harmonics because

$n_{S}$ varies with the harmonics and increases with the poloidal mode number $m$, even though this increase was found to be very small for some parameters (e.g., $\lambda_{m n}$ ). The numbers quoted in the table are essentially those for the higher harmonics. For example, a PCA of $R_{00}$ profile found only 3 significant PC's (accounting for over $99.9 \%$ of the variance in the original data); for $R_{3,5}$ 4 PC's were needed, while 6 PC's were required for $R_{6,6}$. For $B_{m n}$, however, the corresponding number of PC's varied only from 4 for $B_{00}$ to 5 for $B_{6,6}$. For $\lambda_{m n}$ profile, we found that 6 PC's described $99.99 \%$ variance of $\lambda_{00}$, while $\lambda_{6,6}$ needed 7 PC's. 


\begin{tabular}{||c|c||}
\hline \multicolumn{1}{||c||}{ Table 1: Values of $n_{S}$ used for output profile parameters } \\
\hline Regressed parameter & Value of $n_{S}$ \\
\hline$t$ & 4 \\
$B_{m n}$ & 5 \\
$R_{m n}$ & 6 \\
$Z_{m n}$ & 6 \\
$\lambda_{m n}$ & 7 \\
\hline
\end{tabular}

The significance of this number of PC's in the FP model is that it estimates the order of the radial polynomial modelling the profile variable, if that approach had been taken. Thus $B_{00}\left(r_{e f f}\right)$ would have required at least a cubic polynomial in $r_{e f f}$, while $R_{6,6}$ would be poorly recovered by a polynomial lower than 5th order. The FC's for $R, Z$ and $\lambda$, especially those with the poloidal mode number $m>2$, have a more complex radial behaviour than $t$ and $B_{m n}$ and, as such, would need a polynomial of a higher order. Referring to the discussion in the beginning of section 4 , we have thus provided further evidence against the conventional radial polynomial approach.

Figure 7 shows the error profiles for $t$-recovery using c-FP (solid lines) and q-FP (dashed lines) or various values of measurement noise. The X-axis shows $\rho_{\text {eff }}$, which is the normalized $r_{e f f}$, or $\sqrt{s}$. The c-FP recovery errors are significantly smaller for low levels of noise, but from $\sim 20 \%$ noise onwards the two models show similar performance. The ordinate shows the percentage spread (recovery) error defined as

$$
E_{p c}^{(m o)}=100\left(E_{r m s}^{(m o)} / \sigma\right)
$$

for a model $m o \in$ $\{c, q\}$, where $c$ denotes the cubic-FP and $q$ the quadratic-FP model. The quantity $\sigma$ is the spread in the data about the mean value in the database, while $E_{r m s}^{(m o)}$ is the rms error for the model mo. In the context of the output profiles being regressed, both $\sigma$ and $E_{r m s}^{(m o)}$, and therefore $E_{p c}^{(m o)}$, are func- Figure 7: Profiles of recovery errors of iota regression for different tions of $\rho_{\text {eff }}$.

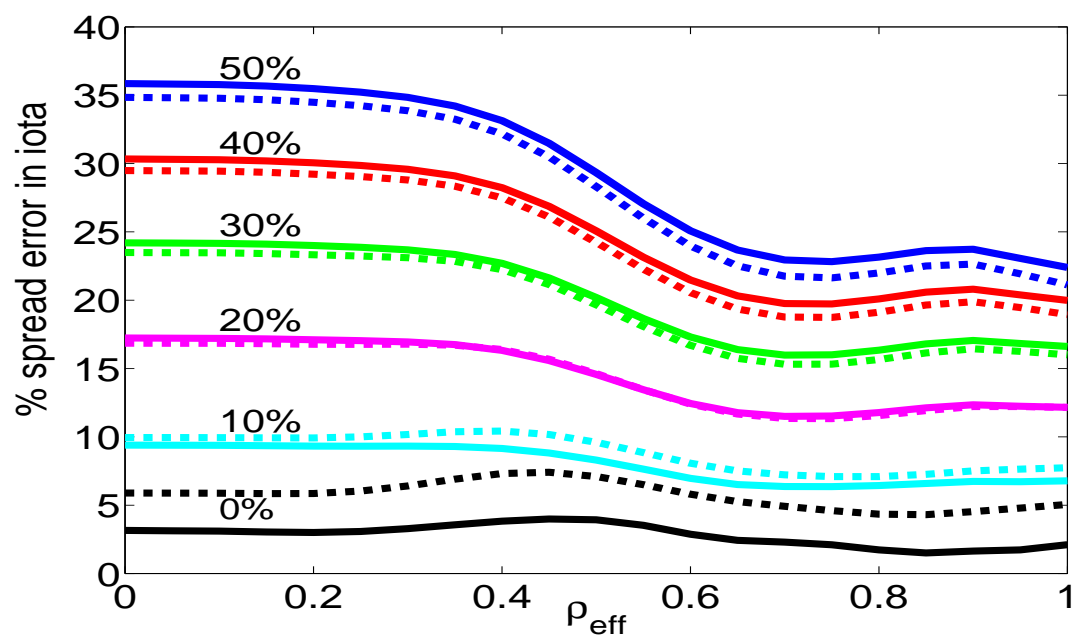

The two sets of plots in figure 8 show the percentage error for the recovery of central- and edge- $t$ as a function of percentage measurement error, where the noise scan on $J(s)$ is quoted along the abscissa. The set of curves plotted in dots are for the recovery of central- $t$. Once again we find that for low levels of measurement noise c-FP (blue dots) is clearly the better model, but its difference with q-FP (red dots) decreases as the noise increases. At 15\%, the blue and 
red dots coincide, before the q-FP curve goes below the c-FP curve. Thus, as in figure 7, the initial superiority of c-FP weakened and finally reversed. However, even at the highest chosen noise level, c-FP is only very slightly "worse" than q-FP.

The set of curves plotted in ' + ' describe the results for edge- $t$ recovery, with blue denoting the c-FP model and red the q-FP model. The blue and the red curves meet around the same value of measurement noise as the dotted curves. Another similarity between the two sets of plots is the significantly larger central- $t$ error compared

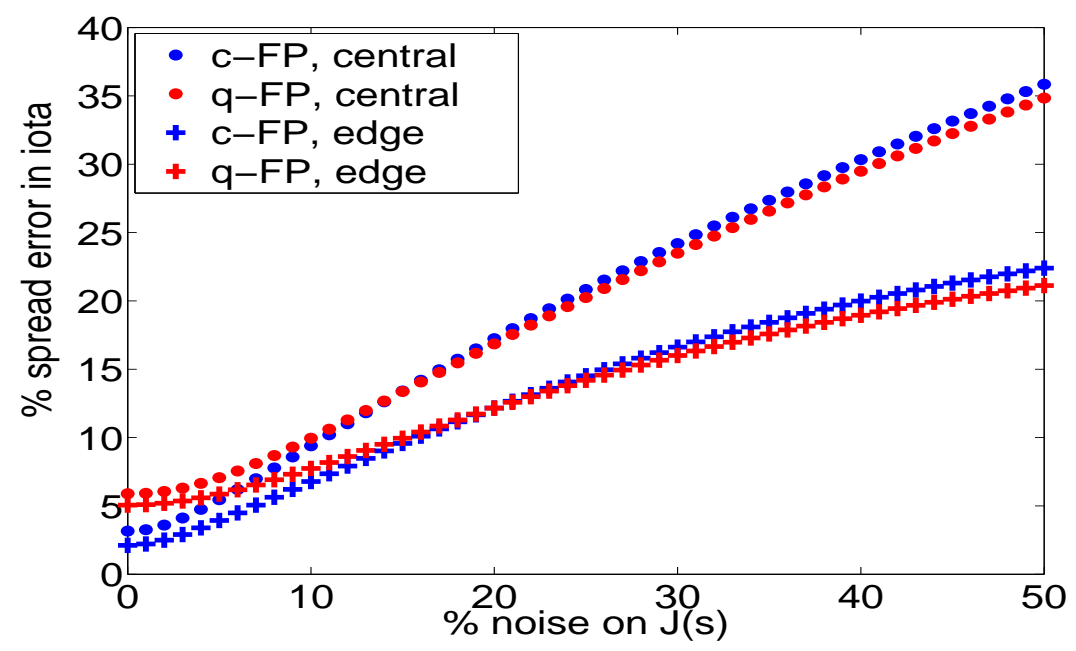

Figure 8: Central- and edge-iota recovery error as a function of measurement noise, with noise on J-profile quoted on abscissa. to the edge- $t$ error for the entire noise scan, and also for noiseless predictors.

For noiseless predictors the larger central- $t$ error implies two possible causes: (a) a larger spread (about the mean) of central- $t$ compared to edge- $t$ values in the database, and (b) a larger variation of central, compared to the edge, current density. By virtue of figure 6 possibility (b) is ruled out, while (a) is true by database construction and we conclude that this is the cause of the observation in discussion.

\begin{tabular}{||c|c|c|c|c|c|c|c||}
\hline \multicolumn{1}{||c|}{ Table 2: Recovery statistics for leading order $R_{m n}$ and $Z_{m n}$} \\
\hline Parameter & $\rho_{\text {eff }}$ & \multicolumn{2}{|c|}{ noise $=0$} & \multicolumn{2}{|c|}{ noise $=20 \%$} & \multicolumn{2}{|c|}{ noise $=50 \%$} \\
\hline & & $E_{p c}^{c}$ & $E_{p c}^{q}$ & $E_{p c}^{c}$ & $E_{p c}^{q}$ & $E_{p c}^{c}$ & $E_{p c}^{q}$ \\
\hline \multirow{3}{*}{$R_{00}$} & 0.0 & 2.99 & 6.82 & 7.60 & 9.16 & 14.74 & 14.98 \\
& 0.5 & 1.84 & 4.56 & 3.82 & 5.48 & 7.79 & 8.27 \\
& 1.0 & 1.59 & 3.28 & 4.37 & 5.04 & 7.97 & 7.87 \\
\hline \multirow{3}{*}{$R_{01}$} & 0.0 & 3.91 & 9.55 & 9.03 & 11.80 & 16.82 & 17.72 \\
& 0.5 & 3.39 & 7.02 & 10.16 & 11.21 & 18.91 & 18.29 \\
& 1.0 & 4.05 & 6.49 & 26.72 & 25.48 & 49.77 & 45.83 \\
\hline \multirow{3}{*}{$R_{02}$} & 0.0 & 5.29 & 9.83 & 7.39 & 10.92 & 12.21 & 14.22 \\
& 0.5 & 6.46 & 12.59 & 14.04 & 17.22 & 22.16 & 24.91 \\
$Z_{01}$ & 1.0 & 12.29 & 17.11 & 22.55 & 24.93 & 36.34 & 34.01 \\
\hline & 0.0 & 4.19 & 9.57 & 7.44 & 10.80 & 13.88 & 14.78 \\
& 0.5 & 3.41 & 6.71 & 7.84 & 9.29 & 14.06 & 14.03 \\
& 1.0 & 4.32 & 8.45 & 23.96 & 23.58 & 43.96 & 40.93 \\
\hline
\end{tabular}

In presence of measurement noise the uncertainty in the edge- $t$ value is basically determined by that in the estimation of the total plasma current $I_{p}$ and the minor radius $a_{\text {eff }}$ of the plasma, the noise in plasma pressure at the boundary being zero as the pressure itself is zero there. Since 
$I_{p}$ is accurately measurable, estimation of $a_{e f f}$ remains the only source of uncertainty on which the error in edge- $t$ effectively depends. On the other hand, the estimation of central- $t$ suffers from errors in the central value of $J(s)$ (and $p(s)$, though its effect may not be pronounced for $t$-recovery). Furthermore, the gain achieved in the plasma edge in the form of reduction of noise level in $I(s)$ (as described in section 5 and figure 5) is absent in the central region. Thus, the central- $t$ is significantly more noisy than the edge- $t$.

To recover the flux surface geometry, we first regressed the FC's $R_{m n}$ and $Z_{m n}$ over the entire profile. Table 2 shows the recovery statistics $\left(E_{p c}^{c}\right.$ and $E_{p c}^{q}$ as defined in eqn (8)) for the leading order $R_{m n}$ and $Z_{m n}$ at three chosen points along the profile - the magnetic axis, the halfway point and the plasma boundary - for each of the noise levels $0,20 \%$
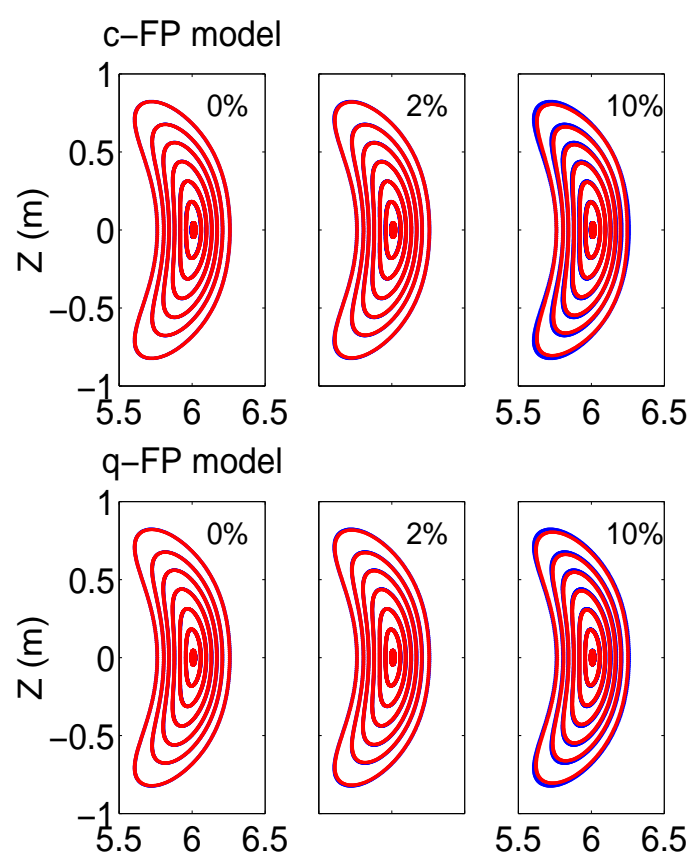
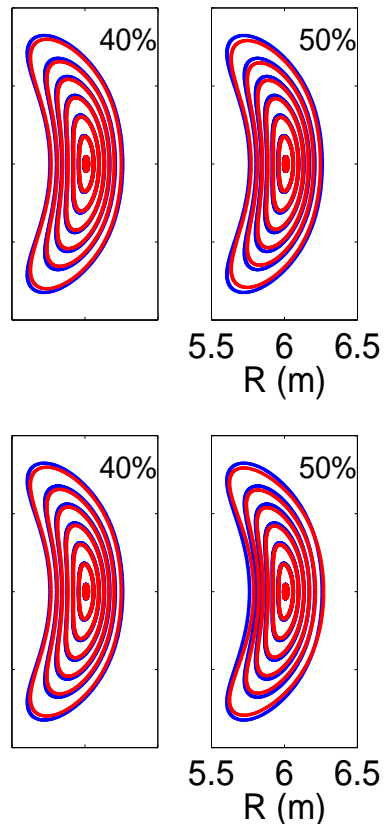
and $50 \%$. For $R_{00}$ we found that at zero noise, Figure 9: Flux surface recovery in the bean-shaped plane for equilibthe percentage spread error decreases monorium case 6385 in the database. Quoted are percentage noise on $J(s)$. Blue: VMEC surfaces; Red: FP-recovered surfaces.

tonically from the axis to the edge, but at higher noise, it decreases from the axis up to $\rho_{\text {eff }}=0.7$ or 0.75 , before increasing again. Even at $20 \%$ measurement noise c-FP is clearly the better model, though at $50 \%$ the two models are comparable. This is something that we also observed for other low order $R_{0, n}, n=1,2$ and 3.

For $Z_{m n}$-recovery some general observations include the following:-

(a) The edge value of at least the low order $Z_{0, n}$ are very sensitive to noise.

(b) For $m>0, Z_{m n}$ error decreases monotonically from the plasma core towards the edge. This is due to fact that the values of $Z_{m n}$, as well as their standard deviations, starting from zero on the magnetic axis, are very small in the core region and monotonically increase towards the plasma edge, so the percentage spread error in the core will tend to be large, and fall off towards the edge.

Next, the FC's were combined in a Fourier series of the forms

$$
R\left(r_{e f f}, u, v\right)=\sum_{m=0}^{M} \sum_{n=-N}^{N} R_{m n}\left(r_{e f f}\right) \cos (2 \pi(m u-n v))
$$




$$
Z\left(r_{e f f}, u, v\right)=\sum_{m=0}^{M} \sum_{n=-N}^{N} Z_{m n}\left(r_{e f f}\right) \sin (2 \pi(m u-n v))
$$

Here $u$ is a poloidal angular coordinate $(0 \leq u \leq 1)$ and $v$ is a toroidal angular coordinate $\left(0 \leq v \leq N_{p}\right)$. For W7-X, $N_{p}$ $=5$, the number of toroidal periods of field and geometry. We also found $M=|N|=6$ sufficient to construct the flux surfaces from the FC's.

Figure 9 shows the bean-shaped cross section of the W7-X flux surfaces on the $v=0$ plane, for one randomly chosen case in the test dataset. The VMEC flux surfaces are shown in blue, while the FP-recovered surfaces are in red. The upper panel shows the c-FP recovery, while the lower one corresponds to q-FP. The re-
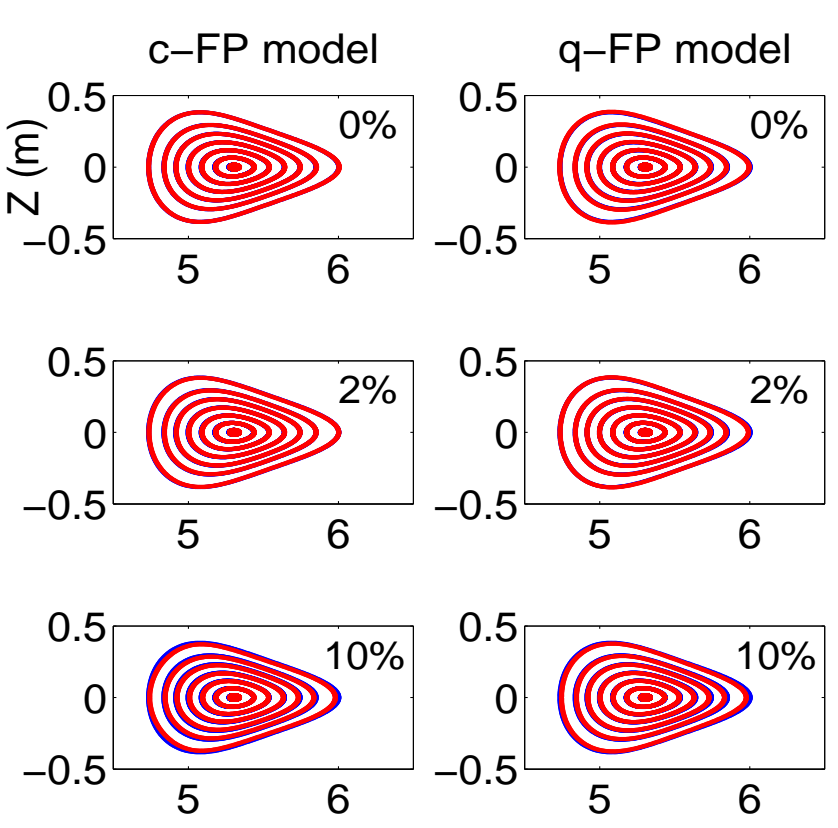
covered flux surfaces compare well with the observed ones up to $10 \%$ noise. A positive aspect of the flux surface recovery is the fitting of the indentation. Above
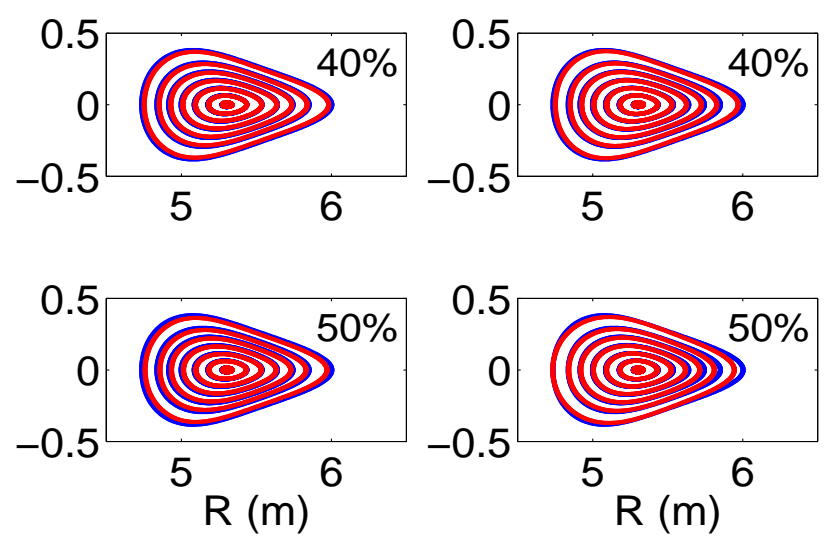

10\% measurement noise, the re- Figure 10: Flux surface recovery in the triangular plane covered surfaces start deviating for equilibrium case 6385 in the database. Quoted are perfrom the observed surfaces. At centage noise on J(s). Blue: VMEC surfaces; Red: FPlarge noise levels q-FP reproduces recovered surfaces.

the points at maximum and min-

imum $Z$ better than c-FP, but not the indentation which is fitted very well by c-FP throughout the noise scan.

Figure 10 shows the comparison of the VMEC flux surfaces with the FP-recovered surfaces in the triangular plane for $v=0.5$, for the same observation as plotted in figure 9 . The tip of the triangular cross section on the outboard side shows a greater sensitivity to noise, as the deviations start from there at $\leq 10 \%$ noise, and this shows up more in the q-FP (compared to c-FP) recovery at large noise levels. Even then, the flux surfaces on the inboard side are well fitted by q-FP, while c-FP-recovered surfaces show a more or less uniform deviation on the inand outboard sides. 


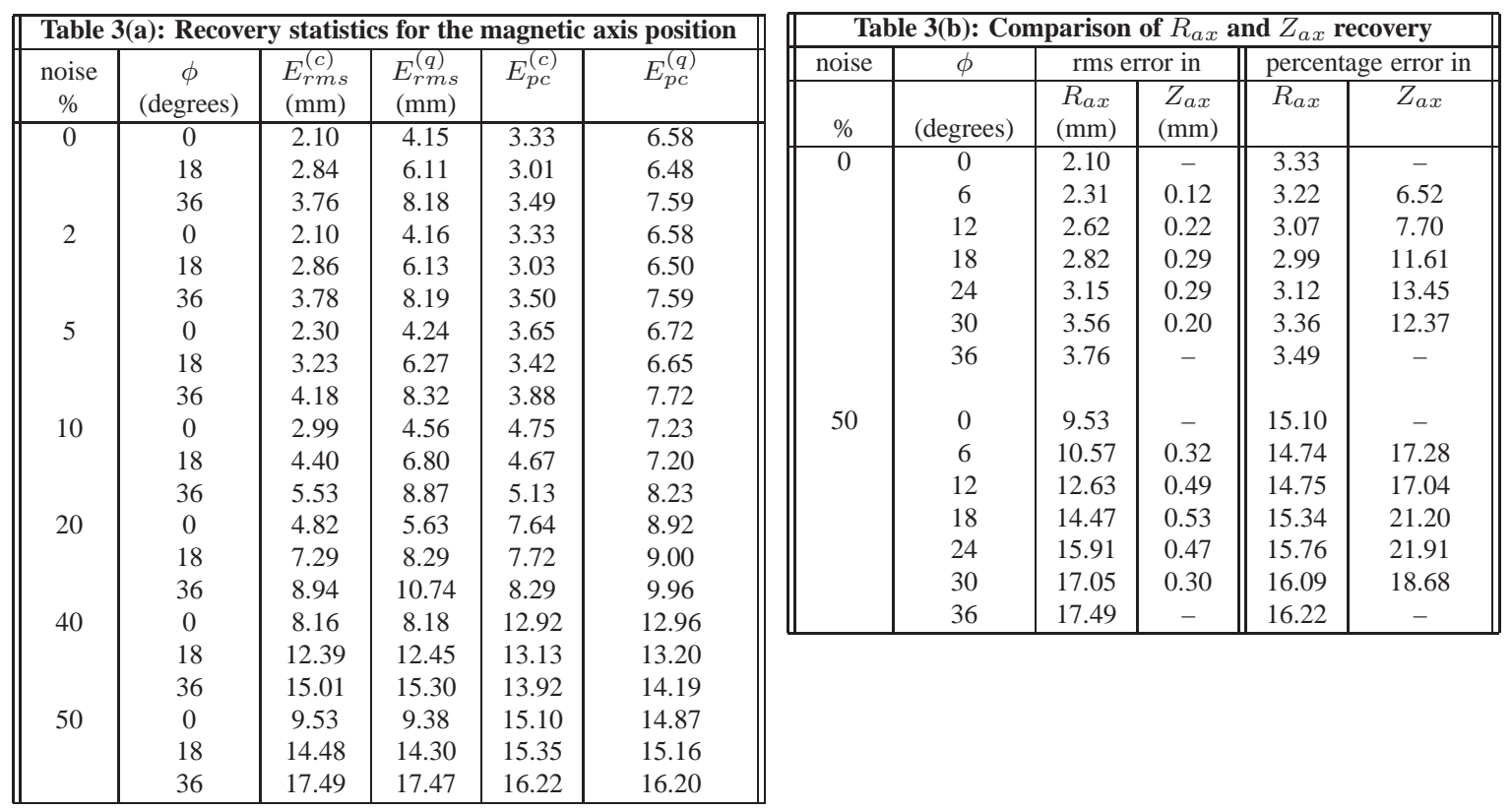

The details of the recovery of the magnetic axis location for $\phi=0,18$ and 36 degrees and different noise levels are listed in Table 3(a), where the toroidal angle $\phi$ is related to $v$ in (9) and (10) by $\phi=2 \pi v / N_{p}$. Here "magnetic axis position" implies its resultant position, i.e., resultant of its $R$ and $Z$ components. We observe that up to $20 \%$ noise, c-FP remains the significantly better model. At the highest noise level, the two models perform similarly.

Table 3(b) compares the recovery of the horizontal $\left(R_{a x}\right)$ and vertical component $\left(Z_{a x}\right)$ of the magnetic axis for the two extreme values of the chosen noise levels. We observe that the percentage spread recovery errors of the radial component are smaller than those of the vertical component even though the absolute errors of $Z_{a x}$ are almost negligible. Note, in Table 3(b), that the $Z$-statistics are not defined on the symmetry planes at $\phi=0$ and 36 , because $Z_{a x}=0.0$.

A quantitative estimate of the quality of flux surface recovery is tabulated in Table 4. The recovery statistic is a root-mean-square (rms) deviation $\left(\delta_{r m s}^{c}\right.$ and $\delta_{r m s}^{q}$ for c-FP and q-FP, respectively) (in unit of length) of the recovered surface from the observed. This was calculated for 20 flux surfaces along the profile. However, results for only the surfaces at $\rho_{\text {eff }}=0.05,0.5$ and 1.0, on the $\phi=0$ plane, are shown in the table. The deviation of the recovered surface from the observed is obtained by dividing the area of the non-overlapping region between the two contours (as seen in a poloidal cross section) by the circumference. The rms deviation is over all observations.

\begin{tabular}{|c|c|c|c|c|c|c|c|c|c|c|c|c|}
\hline \multirow[b]{2}{*}{$\rho_{e f f}$} & \multicolumn{2}{|c|}{ noise $=0$} & \multicolumn{2}{|c|}{ noise $=2 \%$} & \multicolumn{2}{|c|}{ noise $=5 \%$} & \multicolumn{2}{|c|}{ noise $=10 \%$} & \multicolumn{2}{|c|}{ noise $=20 \%$} & \multicolumn{2}{|c|}{ noise $=50 \%$} \\
\hline & $\delta_{r m s}^{c}$ & $\delta_{r m s}^{q}$ & $\delta_{r m s}^{c}$ & $\delta_{r m s}^{q}$ & $\delta_{r m s}^{c}$ & $\delta_{r m s}^{q}$ & $\delta_{r m s}^{c}$ & $\delta_{r m s}^{q}$ & $\delta_{r m s}^{c}$ & $\delta_{r m s}^{q}$ & $\delta_{r m s}^{c}$ & $\delta_{r m s}^{q}$ \\
\hline & $\mathrm{mm}$ & $\mathrm{mm}$ & $\mathrm{mm}$ & $\mathrm{mm}$ & $\mathrm{mm}$ & $\mathrm{mm}$ & $\mathrm{mm}$ & $\mathrm{mm}$ & $\mathrm{mm}$ & $\mathrm{mm}$ & $\mathrm{mm}$ & $\mathrm{mm}$ \\
\hline 0.05 & 0.04 & 0.05 & 0.11 & 0.11 & 0.26 & 0.24 & 0.49 & 0.48 & 0.92 & 0.89 & 1.75 & 1.64 \\
\hline 0.50 & 0.18 & 0.26 & 1.07 & 1.01 & 2.59 & 2.43 & 4.94 & 4.76 & 9.17 & 8.90 & 17.43 & 16.31 \\
\hline 1.00 & 0.31 & 0.41 & 2.09 & 1.96 & 5.08 & 4.79 & 9.74 & 9.39 & 18.11 & 17.58 & 34.44 & 32.25 \\
\hline
\end{tabular}


The results show the following:

a) Except for the noiseless case, the rms deviation increases linearly with radius from the plasma core to the edge.

b) Corresponding to $\rho_{\text {eff }}=0.05,0.5$ and 1.0 , the database (unnormalized) $r_{\text {eff }}$ values have a spread (in $\mathrm{mm}$ ) of $2.47,24.75$ and 49.53, respectively, and rms values (in mm) of 25.15, 251.54 and 503.13, respectively; thus, on the basis of a), the percentage error remains constant over the profile for any non-zero noise level.
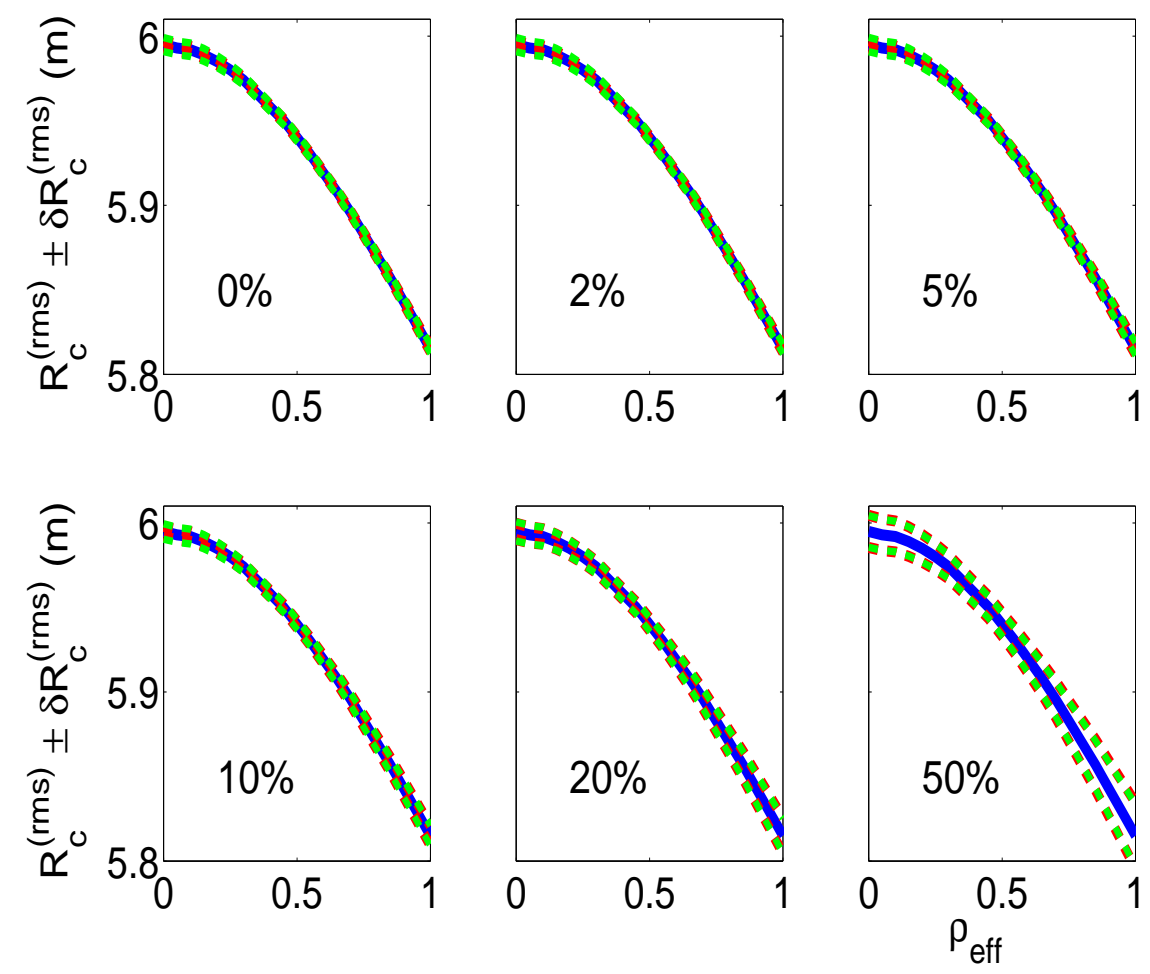

Figure 11: Variation of the (rms value of the) locations, and their "error bars", of the magnetic axis and the flux surface centres on the symmetry plane $\phi=0$. Percentage values give the input noise. Blue curve: Database rms value of $R_{c}$; Red curve: $R_{c}^{(r m s)} \pm \delta R_{c}$ from c-FP recov-

c) For the noiseery; green curve: $R_{c}^{(r m s)} \pm \delta R_{c}$ from $q$-FP recovery. less case, the flux surface at $\rho_{e f f}=0.05$ was recovered with an error of $1.6 \%$, the one at $\rho_{e f f}=0.5$ had a recovery error of $0.73 \%$, while at $\rho_{e f f}=1.0$ the error was $0.63 \%$, suggesting a progressively more accurate recovery towards the plasma boundary.

d) For low noise levels, and up to $10 \%$, the percentage error increases linearly with noise level.

e) The c-FP and the q-FP models perform similarly (in fact, the latter performs slightly better) at all (non-zero) noise levels; the former is significantly better only for the recovery with noiseless predictors.

Some more insight for the geometrical accuracy of the FP can be gained by studying the noise-behaviour of the recovery of the flux surface centres $R_{c}$. For each flux surface along the profile, $R_{c}$ is defined as

$$
R_{c}=0.5[R(\theta=0)+R(\theta=180)]
$$

Figure 11 describes, for the $\phi=0$ plane, the variation of $R_{c}^{(r m s)}$ with $\rho_{e f f}$, where $R_{c}^{(r m s)}$ is the database rms value $R_{c}$. The variation shows the extent of the relative shift of the flux surface centres with respect to the magnetic axis, the location of which is the zero of the abscissa where $R_{c}\left(\rho_{\text {eff }}=0\right)=R_{a x}$. 
The profile of $R_{c}^{(r m s)}$ is plotted in blue in the figure. The figure also plots $R_{c}^{(r m s)} \pm \delta R_{c}^{(r m s)}$, where $\delta R_{c}^{(r m s)}$ is the absolute root-mean-square recovery error of $R_{c}$. The curves corresponding to the c-FP model are in red and those for the q-FP model are plotted in green. The quantity $R_{c}^{(r m s)} \pm \delta R_{c}^{(r m s)}$ is a kind of a confidence interval for $R_{c}^{(r m s)}$ and gives an estimation of an "error bar", though the actual confidence interval will be described if $\delta R_{c}^{(r m s)}$ is replaced by at least one standard deviation of $R_{c}^{(r m s)}$.

Figure 11 shows that the curves are basically indistinguishable up to $20 \%$ noise level, when the errors are of the order of $1 \mathrm{~mm}$. The "error bar" widens at $20 \%$, particularly towards the plasma edge. At $50 \%$ noise we get a significant error range throughout the profile, though the error magnitude remains larger in the edge region (about $1.5 \mathrm{~cm}$ ) than in the core (about $0.6 \mathrm{~cm}$ ). Note, however, that the c-FP and
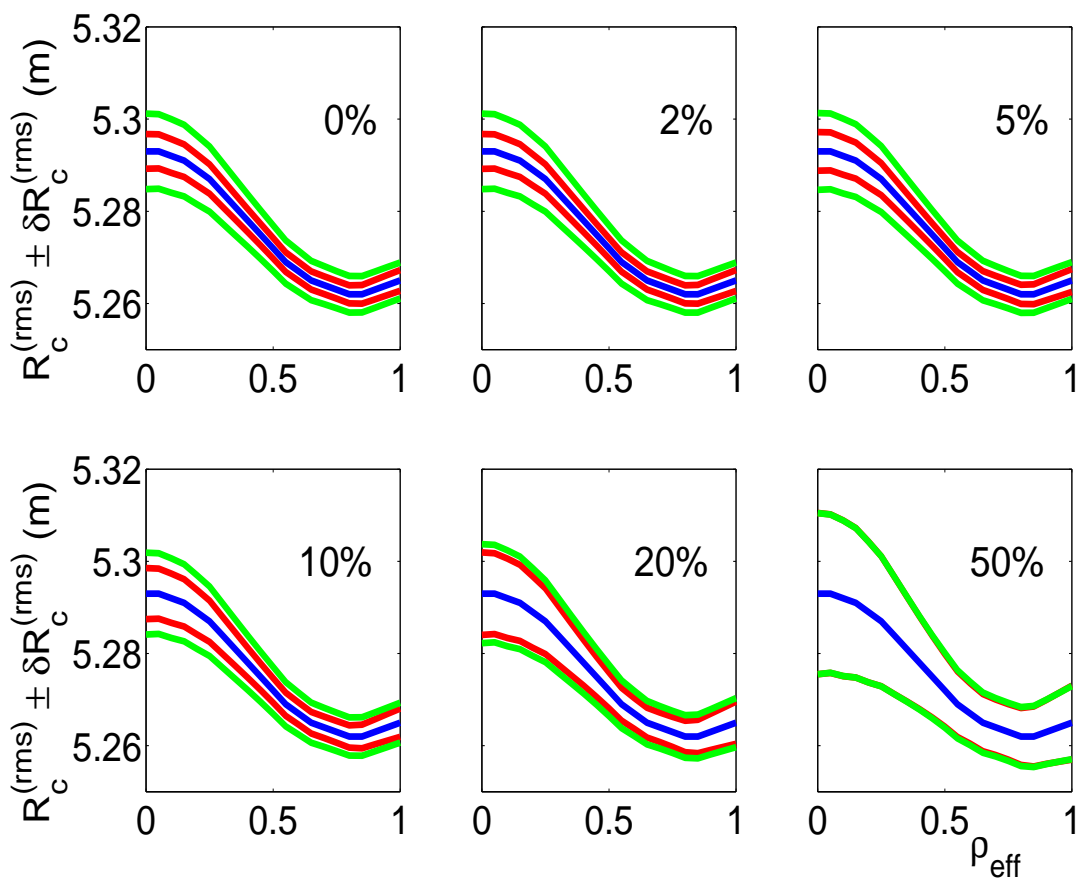
q-FP errors are virtually equal at large noise levels, though Figure 12: Variation of the (rms value of the) locations, and their the former is significantly less for low noise.

"error bars", of the magnetic axis and the flux surface centres on the symmetry plane $\phi=36$. Percentage values give the input noise.

Figure 12 shows similar variations as figure 11 , Blue curve: Database rms value of $R_{c}$; Red curve: $R_{c}^{(r m s)} \pm \delta R_{c}$ from c-FP recovery; green curve: $R_{c}^{(r m s)} \pm \delta R_{c}$ from $q-F P$ recovery. but for the $\phi=36$ plane. We tried to improve the resolution of the curves by expanding the ordinate scale as much as possible. Given that (Radial extension at $\phi=0) /($ Radial extension at $\phi=36) \leq 1 / 2$ we find that the errors on the $\phi=36$ plane are generally smaller than the corresponding errors on the $\phi=0$ plane.

For the recovery of the magnetic field $B\left(r_{e f f}, \theta, \phi\right)$ the same procedure as for the geometry was followed. The magnetic field FC's $B_{m n}\left(r_{e f f}\right)$ were first regressed and then summed according to equation (9). During the summation statistical errors in the FC's may either cancel out or accumulate, so the errors in the reconstruction of $B\left(r_{e f f}, \theta, \phi\right)$ can be quite different from those in the individual $B_{m n}$. Nevertheless, we quote some results from the $B_{m n}$ profile regression, for the low order FC's only, with the FP models. Table 5 shows the (c-FP and q-FP) percentage recovery errors of $B_{00}, B_{01}, B_{02}$ and $B_{11}$ for three positions along the profile - the magnetic axis, the half-way point and the plasma boundary - for each chosen noise level 0, 20\% and 50\%. Note that $B_{11}$ itself vanishes for $\rho_{e f f}=0$ (as do all $B_{m n}$ 's, $m>0$ ), so the percentage errors are quoted for $\rho_{\text {eff }}=0.05$. 


\begin{tabular}{||c|c|c|c|c|c|c|c||}
\hline \multicolumn{6}{||c|}{ Table 5: Recovery statistics for leading order $B_{m n}$} \\
\hline Parameter & $\rho_{e f f}$ & \multicolumn{2}{|c||}{ noise $=0$} & \multicolumn{2}{c||}{ noise $=20 \%$} & \multicolumn{2}{c||}{ noise $=50 \%$} \\
\hline & & $E_{p c}^{c}$ & $E_{p c}^{q}$ & $E_{p c}^{c}$ & $E_{p c}^{q}$ & $E_{p c}^{c}$ & $E_{p c}^{q}$ \\
\hline \multirow{3}{*}{$B_{00}$} & 0.0 & 1.37 & 1.61 & 3.37 & 4.86 & 6.94 & 9.11 \\
& 0.5 & 1.26 & 1.56 & 1.52 & 1.98 & 2.44 & 3.21 \\
& 1.0 & 1.21 & 1.52 & 1.22 & 1.50 & 1.27 & 1.50 \\
\hline \multirow{3}{*}{$B_{01}$} & 0.0 & 0.89 & 1.19 & 1.67 & 1.82 & 2.89 & 2.88 \\
& 0.5 & 0.80 & 0.86 & 1.18 & 1.26 & 1.71 & 1.69 \\
& 1.0 & 0.83 & 0.75 & 2.01 & 1.92 & 3.40 & 3.12 \\
\hline \multirow{3}{*}{$B_{02}$} & 0.0 & 0.85 & 1.26 & 0.90 & 1.40 & 1.35 & 1.68 \\
& 0.5 & 0.80 & 1.09 & 0.89 & 1.18 & 1.21 & 1.41 \\
$B_{11}$ & 1.0 & 0.80 & 0.98 & 1.88 & 1.92 & 3.23 & 3.02 \\
\hline & 0.05 & 2.24 & 4.33 & 25.20 & 24.23 & 47.57 & 44.13 \\
& 0.5 & 1.69 & 3.14 & 24.53 & 23.50 & 46.31 & 42.96 \\
& 1.0 & 1.63 & 2.90 & 19.26 & 18.39 & 36.24 & 33.57 \\
\hline
\end{tabular}

From the table we find the following:-

For $B_{00}$

(a) the error decreases monotonically from axis towards the edge for all levels of noise, but the decrease becomes increasingly sharper as the noise level is ramped up;

(b) the noise sensitivity also has a "profile", in that it varies remarkably along the profile. It is observed that $B_{00}$ is strongly sensitive to noise on the axis, and this sensitivity reduces sharply along the profile so that $B_{00}$ is virtually insensitive to noise on the edge;

(c) the c-FP model produces significantly better results than q-FP throughout the noise scan, even at high noise levels;

For $B_{01}$

(a) at $0 \%$ noise, the error decreases towards the edge, but for c-FP it just flattens after $\rho_{\text {eff }}=0.5$; For q-FP, the flattening is not so pronounced;

(b) the two models are comparable at high noise values; for small noise also, they produce similar errors except in the plasma core region where c-FP outperforms q-FP;

For $B_{11}$

(a) the error monotonically decreases towards the edge; this can again be explained by the small values of $B_{m n}$ around the magnetic axis, and a monotonic increase outwards along the profile;

(b) sensitivity to noise is clearly seen. 


\begin{tabular}{||c|c|c|c|c|c||}
\hline \multicolumn{6}{|c|}{ Table 6: Recovery statistics for $|B|$ on axis } \\
\hline $\begin{array}{c}\text { noise } \\
\%\end{array}$ & $\begin{array}{c}\phi \\
(\text { degrees })\end{array}$ & $\begin{array}{c}E_{r m s}^{(c)} \\
\left(\text { Tesla x } 10^{-3}\right)\end{array}$ & $\begin{array}{c}E_{r m s}^{(q)} \\
\left(\text { Tesla x } 10^{-3}\right)\end{array}$ & $E_{p c}^{(c)}$ & $E_{p c}^{(q)}$ \\
\hline 0 & 0 & 1.63 & 1.91 & 0.87 & 1.03 \\
& 18 & 1.68 & 1.90 & 0.82 & 0.93 \\
& 36 & 1.78 & 2.03 & 0.65 & 0.74 \\
2 & 0 & 1.64 & 2.15 & 0.88 & 1.15 \\
& 18 & 1.72 & 2.13 & 0.84 & 1.04 \\
& 36 & 1.81 & 2.27 & 0.66 & 0.83 \\
5 & 0 & 2.91 & 3.61 & 1.56 & 1.94 \\
& 18 & 2.87 & 3.55 & 1.40 & 1.73 \\
& 36 & 3.10 & 3.75 & 1.13 & 1.37 \\
10 & 0 & 5.12 & 6.11 & 2.75 & 3.28 \\
& 18 & 5.05 & 5.88 & 2.46 & 2.87 \\
& 36 & 5.17 & 6.11 & 1.89 & 2.23 \\
20 & 0 & 8.89 & 8.45 & 4.76 & 4.54 \\
& 18 & 8.32 & 7.69 & 4.06 & 3.75 \\
& 36 & 8.23 & 7.58 & 3.01 & 2.77 \\
40 & 0 & 14.65 & 14.05 & 7.86 & 7.54 \\
& 18 & 12.19 & 11.63 & 5.94 & 5.67 \\
& 36 & 10.42 & 10.21 & 3.80 & 3.72 \\
50 & 0 & 16.28 & 15.62 & 8.74 & 8.38 \\
& 18 & 13.14 & 12.54 & 6.40 & 6.11 \\
& 36 & 10.69 & 10.44 & 3.90 & 3.81 \\
\hline
\end{tabular}

Table 6 shows the error statistics for mod- $B$ on axis, for $\phi=0,18$ and 36 degrees, and the noise sensitivity of these errors, for c-FP and q-FP models. The experimental significance of this quantity lies in following facts:

a) The value of $B$ on axis at $\phi=0$ is needed to know if the ECRH is on- or off-axis.

b) For Electron Cyclotron Current Drive (ECCD), which has been planned with the use of movable mirrors, the wave will be moved out of the $\phi=0$ plane to introduce the propagation constant $k_{\|}$parallel to $B$, so that the values of $B$ at other $\phi$-planes are also important.

The axis FC's $B_{0, n}$ contribute to mod- $B$ on axis, although the errors in the latter depend on the nature of the correlation of errors in the former. From the table we find that up to $10 \%$ noise level c-FP is the better model, but a cross-over occurs above this level and at $20 \%$ noise the q-FP errors are somewhat smaller.

Figure 13 shows the error variation of the mean magnetic field, averaged over $\theta$, with $\rho_{\text {eff }}$ and measurement noise, for $\phi=0,18$ and 36 degree planes (blue, red and green curves in the figure, respectively). The upper panel shows the results for c-FP-recovered field while the lower panel is for q-FP-recovery.

This $\theta$-averaged mean field is given by

$$
<B>\left(r_{e f f}, \phi\right)=\frac{1}{2 \pi} \int_{0}^{2 \pi} \sum_{m} \sum_{n} B_{m n} \cos \left(m \theta-n N_{p} \phi\right) d \theta
$$

which integrates to 


$$
<B>\left(r_{e f f}, \phi\right)=\sum_{n} B_{0, n} \cos \left(n N_{p} \phi\right)
$$

Thus, only the $m=0$ terms survive and the errors in the leading order $B_{0, n}$ determine those in $\left\langle B>\left(r_{e f f}, \phi\right)\right.$. The error profile
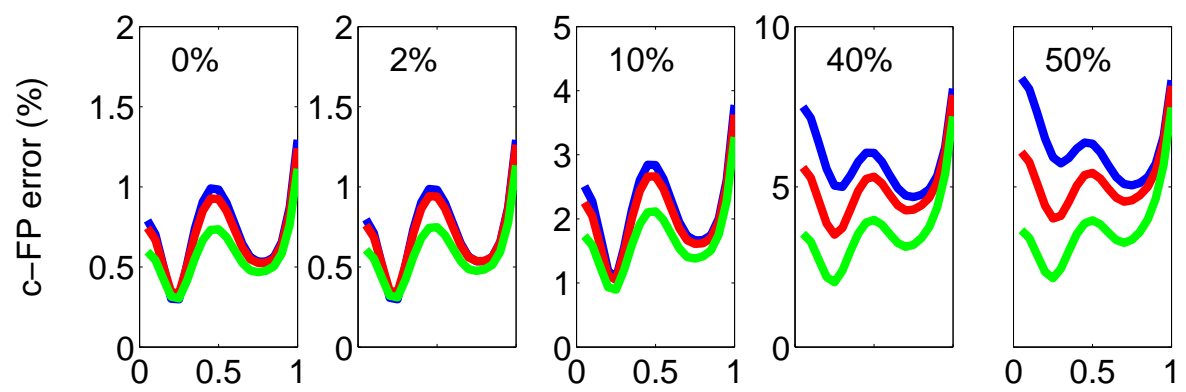

in figure 13 shows the influence of $B_{00}$ as the dominating Fourier component only in certain parts of the profile, namely, the plasma core, the middle $\left(\rho_{\text {eff }}=0.5\right)$
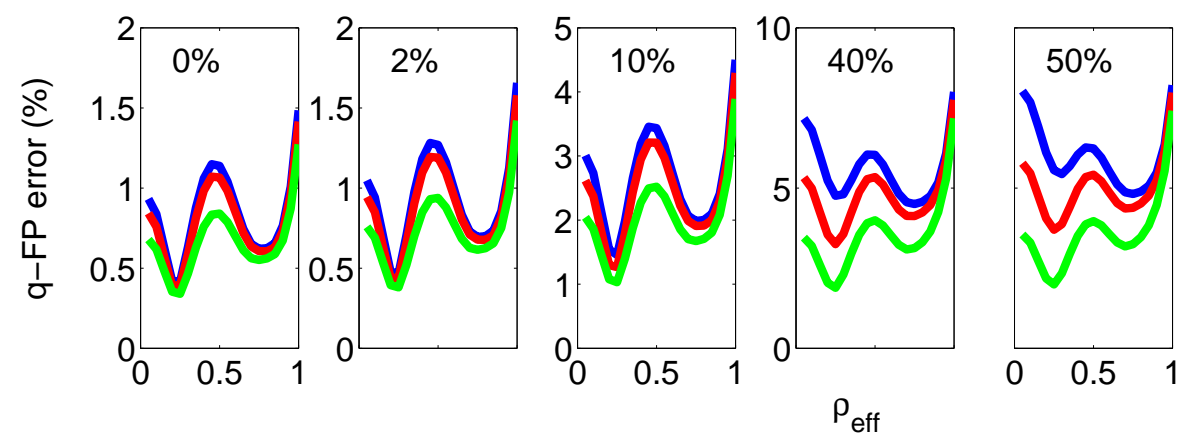
and the edge region. The oscillating error profile shows that in

Figure 13: Variation of percentage spread recovery error of the magnetic field as a function of normalized effective radius as well as measurement noise (given by the percentage numbers on the figures). Error on axis have not been plotted. Blue curve: $\phi=0$; Red curve: $\phi=18$; Green curve: $\phi=36$.

other regions the higher order $B_{0, n}$ also have a strong influence in the recovery of $\theta$-averaged mean field. Note that the error on the axis itself is not shown in figure 13, these being tabulated in table 6. For $\phi=0$ the error is most sensitive to noise, while at $\phi=36$ it shows the least sensitivity except at the plasma edge where both models show similar errors for $\phi=0,18$ and 36 deg, and this similarity is observed for all the noise levels considered. Comparing the two FP models, our general conclusion is that the c-FP model is significantly better than q-FP at low noise levels, while the two models show similar performance at large noise.

Next we look at the recovery of the stream function $\lambda$ which relates to obtaining magnetic coordinates and thus get the field line direction.

\begin{tabular}{||c|c|c|c|c|c|c|c||}
\hline \multicolumn{6}{||c|}{ Table 7: Recovery statistics of leading order $\lambda_{m n}$} \\
\hline Parameter & $\rho_{\text {eff }}$ & \multicolumn{2}{|c||}{ noise $=0$} & \multicolumn{2}{c||}{ noise $=20 \%$} & \multicolumn{2}{c|}{ noise $=50 \%$} \\
\hline & & $E_{p c}^{c}$ & $E_{p c}^{q}$ & $E_{p c}^{c}$ & $E_{p c}^{q}$ & $E_{p c}^{c}$ & $E_{p c}^{q}$ \\
\hline \multirow{3}{*}{$\lambda_{01}$} & 0.0 & 23.77 & 27.35 & 30.24 & 31.91 & 44.09 & 43.28 \\
& 0.5 & 25.12 & 29.31 & 31.16 & 33.15 & 44.53 & 43.62 \\
& 1.0 & 25.77 & 29.36 & 28.13 & 30.85 & 34.13 & 35.03 \\
\hline \multirow{3}{*}{$\lambda_{02}$} & 0.0 & 84.40 & 82.35 & 83.79 & 82.37 & 84.43 & 83.36 \\
& 0.5 & 70.49 & 70.73 & 71.07 & 70.98 & 73.52 & 74.12 \\
& 1.0 & 50.79 & 57.80 & 51.79 & 58.37 & 55.54 & 60.06 \\
\hline
\end{tabular}


Table 7 contains the recovery statistics of the profile of some leading order FC's $\lambda_{m n}$, where the percentage errors from c-FP and q-FP models are quoted at three positions along the profile - the magnetic axis, the plasma boundary and the half-way point. From the large errors (even without measurement noise) it is clear that the recovery was quite challenging.

However, this aspect of the recovery was to be expected to some extent, because for the FC's the recovery has a strong dependency on the spectral minimisation [10] used in the VMEC2000 code. When a Fourier decomposition is spectrally minimised, it means the high order Fourier harmonics are penalized. Spectral minimisation leads to a unique determination of the poloidal angular coordinate. In this context it should be pointed out that while $R_{m n}$ and $Z_{m n}$ are spectrally minimised in VMEC2000, $B_{m n}$ and $\lambda_{m n}$ are not. The latter, therefore, have broader spectra which may lead to larger recovery errors. However, only the recovery of $\lambda_{m n}$ was affected by this problem. The reason possibly lies in the small magnitudes of $\lambda_{m n}$, even those in the leading order, so that FP had difficulties in recovering them accurately.

The FC's were summed up over the Fourier modes according to equation (10) to obtain $\lambda\left(r_{e f f}, \theta, \phi\right)$. If we now turn to Table 8 , we find that the errors of estimation of $\lambda$ on axis follow those for the FC's, i.e., they are also poorly recovered, even without noise. That is why only the statistics for zero noise are shown. It should be noted that $\lambda\left(r_{\text {eff }}, \theta, \phi\right) \sim 0$ on the magnetic axis for $\phi=0,36$.

\begin{tabular}{||c|c|c|c|c|c||}
\hline \multicolumn{7}{||c||}{ Table 8: Recovery statistics of $|\lambda|$ on axis } \\
\hline $\begin{array}{c}\text { noise } \\
\%\end{array}$ & $\begin{array}{c}\phi \\
(\text { degrees })\end{array}$ & $\begin{array}{c}E_{r m s}^{(c)} \\
\left(\text { Radian } 10^{-3}\right)\end{array}$ & $\begin{array}{c}E_{r m s}^{(q)} \\
\left(\text { Radian } \mathrm{X} 10^{-3}\right)\end{array}$ & $E_{p c}^{(c)}$ & $E_{p c}^{(q)}$ \\
\hline \multirow{3}{*}{0} & 6 & 2.10 & 2.03 & 44.83 & 43.22 \\
& 18 & 4.82 & 4.73 & 67.83 & 66.62 \\
& 30 & 3.27 & 3.33 & 50.04 & 59.12 \\
\hline
\end{tabular}

Figure 14 describes the error variation of mean $\lambda$, averaged over $\theta$, with $\rho_{\text {eff }}$ and measurement noise. The $\theta$-averaged $\lambda$ was calculated in the same way as the corresponding quantity for $B$ shown in equations (12) and (13). As in Figure 13, the blue, red and green curves describe the statistics on $\phi=0,18$ and 36 degrees, respectively, and the axis errors are not shown. The errors in the plasma core are generally large, following the pattern on the magnetic axis. The point worth noting here is the sharp decrease of the error outside the core region, typically for $\rho_{\text {eff }} \geq$ 0.2 , where the errors are of the order of only a few percent of the spread. In fact, the decrease becomes even sharper with increasing noise, because the edge error increases only very little compared to the error in the core.

Thus, even though the individual $\lambda_{m n}$ 's were poorly recovered by the FP models, the reconstructed $\lambda\left(r_{e f f}, \theta, \phi\right)$ showed a good recovery accuracy throughout the profile except the plasma core. This might suggest that the large errors in the $\lambda_{m n}$ 's were strongly anti-correlated and therefore (mostly) cancelled out. The results shown in figure 14 also show that the recovery of the magnetic field line direction with the present method was a reasonable success, although some more investigation may be necessary in view of the large $\lambda_{m n}$ errors. 
(b) Sensitivity test of some regressed output parameters.

In our analysis, the parameters describing the physical state of the plasma magnetic configuration were recovered from
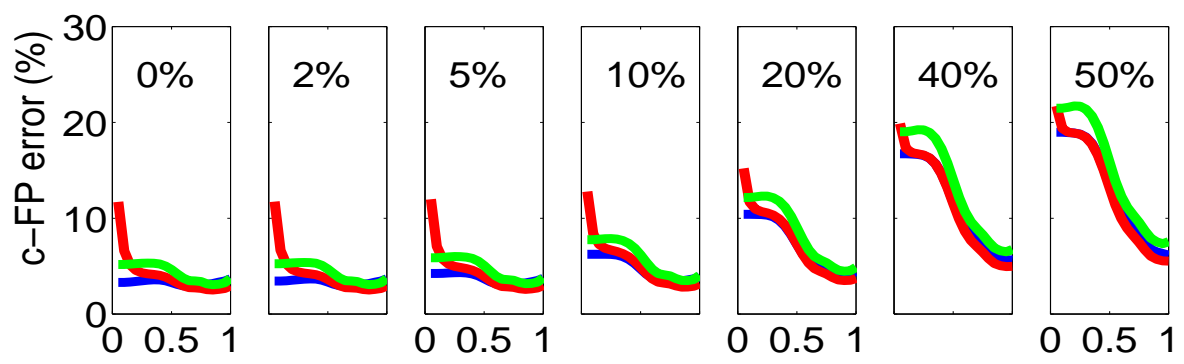

simulated measurements from which the 15 predictors, or inputs, to the statistical model were generated. It would be interest-
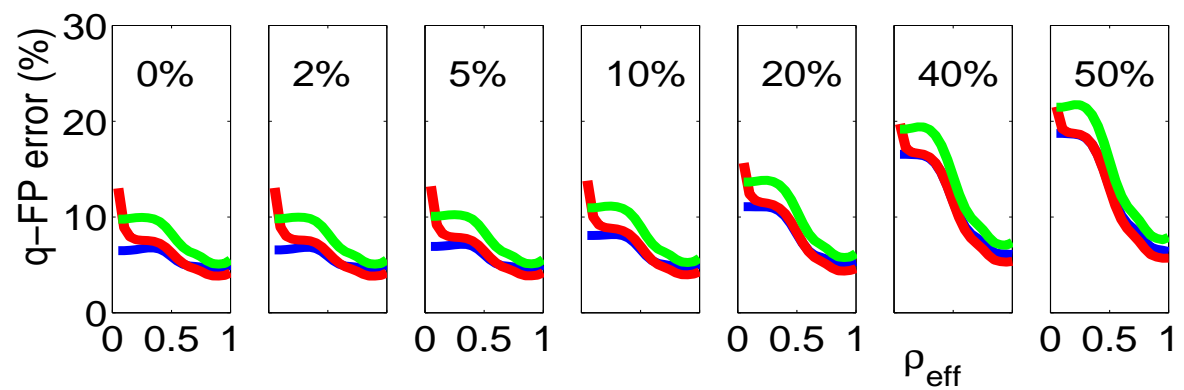

ing to test the spe- Figure 14: Variation of percentage spread recovery error of $\lambda$ as a function cific dependencies of normalized effective radius as well as measurement noise (given by the of a few of the out-percentage numbers on the figures). Error on axis have not been plotted. put parameters re- Blue curve: $\phi=0$; Red curve: $\phi=18$; Green curve: $\phi=36$.

covered from the model on the different basic "measurements". This was done by removing a particular predictor $S_{I}$ from the full set, recovering an output parameter $S_{O}$ and comparing the recovery errors with those obtained for $S_{O}$ with the full set of noiseless inputs, thereby getting an idea of the influence the excluded input $S_{I}$ has on $S_{O}$.

Our basic "measurements" included the external coil currents, $a_{e f f}$, and the distributions of plasma pressure and toroidal plasma current. These formed 6, 1, 4 and 4 predictors, respectively. We chose to exclude a "measurement" by simply reducing the value of the concerned predictor(s) to zero, keeping the predictors for the other "measurements" at their noiseless values. This gives a signal-to-noise ratio of zero for the predictor(s) removed, the percentage noise being infinity, for the excluded measurement. It is to be noted here that this test is only for the sensitivity of the output plasma parameters to the inputs, so the possible correlations of the measurement noise is ignored here.

Figure 15 shows the effect of excluding, in turn, the coil currents, $a_{e f f}$, the plasma current and pressure profile, on the rms error profile of $t$. As before, $\rho_{\text {eff }}$ is the normalised $r_{\text {eff }}$. The figure also plots, for the sake of comparison, the error profile when the entire set of 15 inputs corresponded to ideal, or noiseless measurements (indicated as "All inputs ideal" in the figures), and also when they were all simultaneously excluded from the input set (indicated as "No inputs" in the figures). The latter case, corresponding to a signal-to-noise ratio of zero simultaneously for all the measurements, just plots the profile of the standard deviation in $t$, meaning a $100 \%$ recovery error, and is obviously the worst scenario for the output errors.

The central- $t$ error is the largest when $I(s)$ is removed, because the central- $t$ value strongly 
depends on the central current density. The pressure profile has a substantial effect on central- $t$, though a very small effect on the boundary- $t$. When $a_{e f f}$ was excluded, $t$-profile was moderately affected. The external coil currents also have a strong overall influence which, at the boundary, is even stronger than the $I(s)$ effect.

\section{Conclusions}

Equilibrium reconstruction of W7-X magnetic configuration at finite- $\beta$ using essentially non-magnetic measurements showed excellent recovery accuracy at low levels of measurement noise (added simultaneously to perturb all predictors), usually up to around $10 \%$ on $J(s)$, using a cubic polynomial model. This sup-

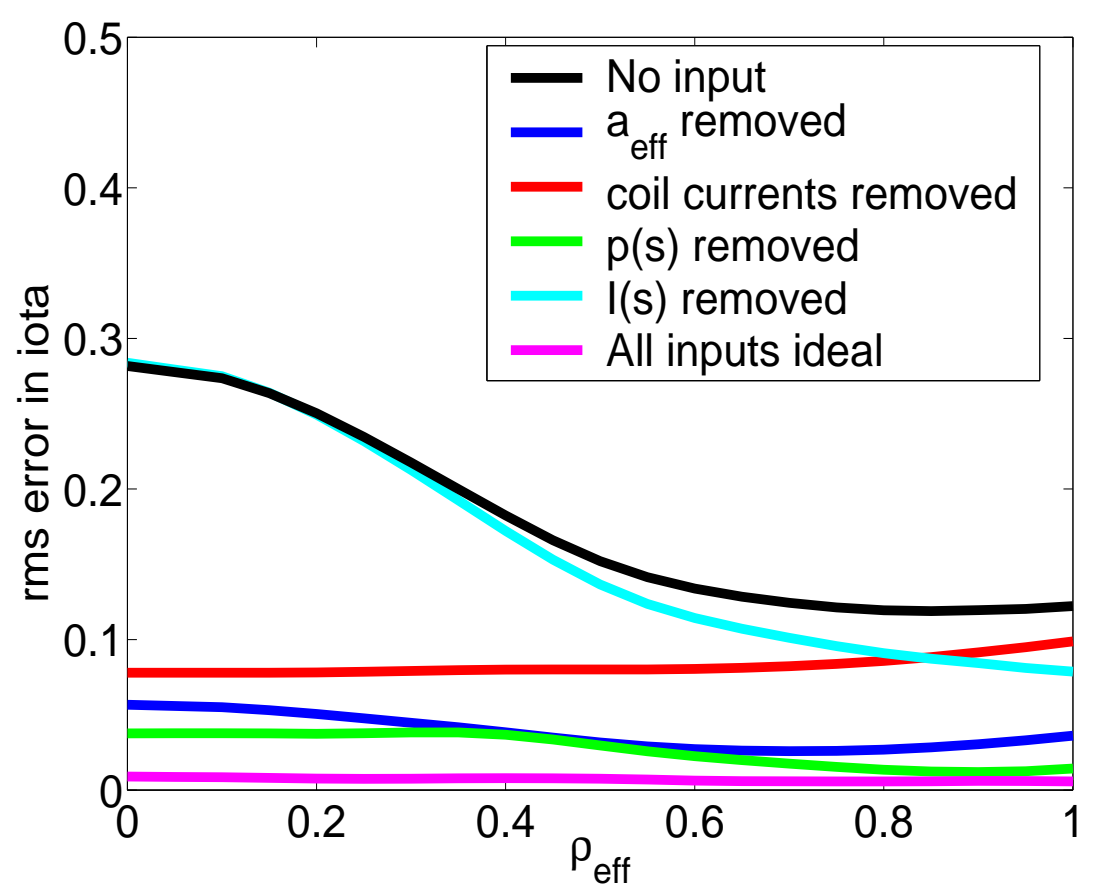

ported earlier results on Figure 15: Studying the influence of the "measurements" on the iota vacuum analysis. The profile recovery. Removal of one measurement means the rest are at results obtained with ex- their exact values. For comparison with the extreme cases, the error act inputs would be very profile for all ideal inputs (magenta curve) and no inputs (black curve) useful in providing fast are also shown.

transformations for diagnostics, thereby avoiding the use of time-consuming equilibrium codes , and may also serve to provide good starting configurations for a more rapid convergence of the equilibrium codes if they are needed.

With increase of measurement noise levels the difference in performance between the cubic and the quadratic polynomials reduced. In the worst case scenario of the chosen noise limits, that corresponded to $50 \%$ of $J(s)$-profile noise, $20 \%$ of pressure profile noise and $10 \%$ noise in $a_{e f f}$, the two models performed similarly. The only exception was the recovery of the flux geometry, where the two models produced errors of similar order for any (non-zero) value of noise.

The recovery of the profiles of $\lambda_{m n}$ produced very large errors, as did the axis recovery of $\lambda$. However, the errors sharply reduced on the flux surfaces outside the plasma core to very small values, to show an impressive reconstruction. The edge values were only mildly affected by measurement errors. Even then, this recovery may need some more investigation in view of the large errors in the individual $\lambda_{m n}$.

However, for all the similarities of its performance with the q-FP model at large noise levels, 
c-FP should still be the recommended model due to its overall reliability. In fact, some of the results suggest that, by using an alternative method to the linear regression, namely, errors-invariables approach $[14,15]$ which is meant exclusively for system identification in presence of noise in predictors, c-FP is likely to perform significantly better even at high noise levels.

The entire analysis was considerably simplified by the use of the PC's of the output profile variables, rather than the conventional radial polynomial approach. This was further demonstrated in the number of significant PC's the different profile variables corresponded to, especially for the Fourier coefficients.

In the second part of the analysis, recovery of the plasma magnetic configuration was studied again by excluding a subset of the predictors from the full set, leaving the rest at their exact values. This is a test of the importance of the excluded predictors, and therefore of the corresponding "measurements", for the recovery of different properties of the magnetic configuration. The test was carried out for the profiles of rotational transform as an example.

The plasma configurations in the database used in this analysis had only nested flux surfaces, and magnetic islands were neglected. Thus, the plasmas were basically limiter bound. To have islands in the analysis, the database needs to be generated with codes such as HINT or PIES which are still too time consuming with strong CPU requests to be used for generating thousands of equilibria.

Essentially non-magnetic measurements were used for this analysis. In the near future, we plan to report work where magnetic measurements, replacing the profile data, would be used to recover the finite- $\beta$ configurations.

\section{Acknowledgments}

This work was funded by a reciprocal research agreement between IPP and UCC. In this respect, authors AS and PJM would like to acknowledge support under the European Union Mobility Programme, and express gratitude to IPP for its hospitality. The authors also extend their sincere thanks to Dr.Andreas Werner of IPP-Greifswald, Germany, for providing information on the measurement errors for the total plasma current, and Dr.Henning Maassberg, also of IPP-Greifswald, who had spared valuable time to read the paper and give critical comments.

\section{References}

[1] Beidler C. et al 1990 Fusion Technol. 17148

[2] Grieger G. et al 1992 Phys. Fluids B 42081

[3] Sengupta A., McCarthy P.J., Geiger J. and Werner A. 2004 Nucl. Fusion 441176

[4] Braams B.J., Jilge W. and Lackner K. 1986 Nucl. Fusion 26699

[5] van Milligen B.Ph. and Lopes Cardozo N.J. 1991 Comput. Phys. Commun. 66243

[6] McCarthy P.J. and Morabito F.C. 1997 Int. J. Appl. Electromag. Mech. 8343

[7] Wagner F. et al 2005 Phys. Plasmas 12072509 
[8] Harafuji K., Hayashi T. and Sato T. 1989 J. Comp. Phys. 81169

[9] Reiman A.H. and Greenside H.S. 1986 Comput. Phys. Commun. 43157

[10] Hirshman S.P., Van Rij W.I. and Merkel P. 1986 Comput. Phys. Commun. 43143

[11] Callaghan H.P., McCarthy P.J. and Geiger J. 1999 Nucl. Fusion 39509

[12] Hirshman S.P. and Whitson J.C. 1983 Phys. Fluids 263553

[13] Mc Carthy P.J., Sengupta A., Geiger J., Werner A. "Statistical Analysis of the equilibrium configurations of W7-X stellarator using Function Parametrization" 15th International Stellarator Workshop, Madrid, Spain (October 3 - 7, 2005) P2-09

[14] Mardia K.V., Kent J.T., Bibby J.M. 1979 Multivariate Analysis, Academic Press, London 189

[15] Kardaun O.J.W.F. 2005 Classical Methods of Statistics, Springer-Verlag Berlin 115 